\title{
Shared Microglial Mechanisms Underpinning Depression and Fatigue and Their Comorbidities
}

Adriano José Maia Chaves Filho ${ }^{1}$, Danielle S. Macedo ${ }^{1,2}$, David Freitas de Lucena ${ }^{1}$, Michael Maes $^{3,4}$

${ }^{1}$ Neuropsychopharmacology Laboratory, Drug Research and Development Center, Faculty of Medicine, Federal University of Ceara, Fortaleza, CE, Brazil; adrianoafilho@hotmail.com (A.J.M.C.F.); daniellesilmacedo@gmail.com (D.S.M.); daviddelucena@me.com (D.F.L.)

${ }^{2}$ National Institute for Translational Medicine (INCT-TM, CNPq), Ribeirão Preto, Brazil ${ }^{3}$ Department of Psychiatry, Faculty of Medicine, Chulalongkorn University, Bangkok, Thailand. ${ }^{4}$ IMPACT Strategic Research Center, Deakin University, Geelong, Australia

Corresponding author:

Prof. Dr. Michael Maes, M.D., Ph.D.dr.michaelmaes@hotmail.com

Abstract: In 2011, it was reviewed that there is a strong co-occurrence between major depression and chronic fatigue syndrome (CFS), with fatigue and physio-somatic symptoms being key symptoms of depression, and depressive symptoms appearing during the course of CFS. Moreover, the comorbidity between both conditions may in part be explained by activated immune-inflammatory pathways, including increased translocation of Gram-negative bacteria and increased levels of pro-inflammatory cytokines, such as interleukin (IL)-1. Nevertheless, the possible involvement of activated microglia in this comorbidity has remained unclear. This paper aims to review microglial disturbances in major depression, CFS and their comorbidity. A comprehensive literature search was conducted using the PubMed / MEDLINE database to identify studies that are relevant to this current review. Depressed patients present neuroinflammatory alterations, probably related to microglial activation, while animal models 
show that a microglial response to immune challenges including lipopolysaccharides is accompanied by depressive-like behaviors. Recent evidence from preclinical studies indicate that activated microglia have a key role in the onset of fatigue. In chronic inflammatory conditions, such as infections and senescence, microglia orchestrate an inflammatory microenvironment thereby causing fatigue. In conclusion, based on our review we may posit that shared immune-inflammatory pathways and activated microglia underpin comorbid depression and CFS and that activated microglia are the main orchestrators of this comorbidity. As such, microglial activation and neuro-inflammation may be promising targets to treat the overlapping manifestations of both depression and CFS.

KEYWORDS: major depressive disorder, microglia, cytokines, neuro-immune, chronic fatigue, oxidative stress

\section{List of Abbreviations:}

CNS - central nervous system; E - embryonic day; PAMPs - pathogen-associated molecular patterns; DAMPs - damage-associated molecular patterns; GABA - gamma aminobutyric acid; ATP - Adenosine 5'-triphosphate; IL - interleukin; TNF - Tumor Necrosis Factor; AMPA - $\alpha$ amino-3-hydroxy-5-methyl-4-isoxazolepropionic acid; NMDA - N-methyl-D-aspartate; LTP long-term potentiation; LTD - long-term depression; LPS - lipopolysaccharide; IFN - interferon. 


\section{Introduction}

Microglial cells are the major myeloid cells of the central nervous system (CNS), comprising $\sim 5-15 \%$ of the glial cells in the brain [1,2]. Accumulating evidence points towards a role of microglia in brain homeostasis and pathological processes [3]. Indeed, under physiological conditions microglia regulate neuronal function and morphology. During neurodevelopment microglia are responsible for "synaptic pruning", while in adult brain, microglia are responsible for regular selection of adult synapses, called "synapse sampling". Under physiological conditions both synaptic pruning and sampling are important to the maintenance of synaptic strength as well as elimination of redundant synapses thereby contributing to synapse development and plasticity [4]. Microglia affect neural circuits through the secretion of several bioactive factors, such as cytokines, chemokines, the gaseous transmitter nitric oxide (NO) or neurotransmitters (ATP and glutamate), and metabolites (for example the tryptophan catabolites kynurenine and quinolinic acid). These neuroactive substances can rapidly modulate neuronal functions, thereby influencing membrane excitability and synaptic strength, through gene expression-dependent mechanisms [4].

Under pathological conditions, microglial activation and the resulting inflammatory response allow the elimination of pathogens and damaged cells [2]. Despite this, in neurodegenerative disorders these cells induce an abnormal secretion of toxic substances, such as reactive oxygen species, cytokines and catabolites, resulting in the elimination of functional synapses [5].

Based on the reciprocal interactions between microglia and neurons, microglial cells have been increasingly studied in the context of mental disorders [6-8]. It is important to highlight that psychosocial stress, a major trigger of stress-related disorders, is known to induce changes in microglia morphology in corticolimbic regions that regulate mood [9]. Therefore, chronic stress exposure causes microglial activation thereby affecting synaptic function, a mechanism related to depression neurobiology [10]. 
In the last decades, more precisely since the 1990s, activated immune-inflammatory pathways, including increased production of interleukin (IL)-1ß, were found in major depression (MDD). In this regard, the first evidence for immune-inflammatory alterations in depression came from preclinical [11] and clinical studies [12]. Since then, preclinical models using the endotoxin from gram-negative bacteria, lipopolysaccharide (LPS), as immune challenge revealed time-related alterations induced by its exposure ranging from sickness behavior (as an early response $\sim 2 \mathrm{~h}$ post LPS) to depression (24 h post LPS) $[11,13]$. More recently, high plasma levels of IgA/lgM responses to sonicated Gram-negative gut commensal bacteria and LPS were observed in MDD and anxious patients confirming the participation of Gram-negative bacteria and their endotoxins in the pathophysiology of affective disorders $[14,15]$. Moreover, signs of increased translocation of Gram-negative bacteria and LPS were significantly associated with activated immune-inflammatory pathways and increased oxidative and nitrosative stress in MDD [16].

Mechanistic explanations to LPS-induced depressive-like alterations are related to the activation of the NOD-like receptor family, pyrin domain containing 3 (NLRP3) inflammasome in microglial cell with a consequent release of pro-inflammatory cytokines such as interleukin (IL)$1 \beta$ [17]. Indeed, these brain pro-inflammatory alterations compromise neuroendocrine and neurotransmission mechanisms, among others, contributing to depression symptomatology and possibly to a future subclassification of depressed patients based on their inflammatory profile [18].

Recently, it was reviewed that there is a strong co-occurrence between MDD and chronic fatigue syndrome (CFS), with fatigue and physio-somatic symptoms being key symptoms of depression, and depressive symptoms appearing during the course of CFS [19]. Furthermore, fatigue is one of the residual symptoms following MDD treatment being related to lack of remission, poorer psychosocial functioning, lower health-related quality of life and a more chronic course of depression [20]. Clinical research has shown that chronic fatigue is 
accompanied by activated immune pathways, including increased IL-1 production, oxidative and nitrosative stress and increased bacterial translocation, as assayed by $\lg \mathrm{A} / \lg \mathrm{M}$ responses to sonicated Gram-negative bacteria $[19,21]$. Increased bacterial translocation in chronic fatigue is accompanied by signs of peripheral inflammation, including increased IL-1 production [21].

Moreover, in MDD, increased levels of IL-1 are associated with higher levels of fatigue and physio-somatic symptoms, including hyperalgesia, insomnia and neurocognitive deficits, suggesting that activated immune-inflammatory pathways (including IL-1-related mechanisms) may underpin the comorbidity between MDD and chronic fatigue [22]. Recent results obtained from animal models of fatigue revealed that this condition is associated with activated microglia and brain release of cytokines and chemokines [23]. In this regard, IL-1 is emerging as a mediator of fatigue [24].

Thus, based on the importance of immune activation and activated microglia in MDD and fatigue and their comorbidity this paper aims to review: i) microglia physiological and pathological mechanisms, ii) microglial disturbances in depression and iii) microglial alterations underlying fatigue and CFS. The results will expand the knowledge on microglia involvement in depression and fatigue and their comorbidity and will provide new insights for a better treatment approach for depression and fatigue based on therapeutic strategies targeting microglial stabilization.

\section{Search strategy}

A comprehensive literature search was conducted with the PubMed/MEDLINE database to identify studies that were relevant to this current review. The search terms "microglia" [MeSH] OR "inflammation" [MeSH] OR "cytokines" [Mesh] OR "interleukins" [Mesh] OR "Tumor Necrosis Factor-alpha" [MeSH] OR "translocator protein (18 kDa)" [MeSH] OR "tryptophan" [MeSH] OR "kynurenine" [MeSH] OR "serotonin" [MeSH] were cross-referenced with "Depression" [MeSH] OR "Depressive Disorder" [MeSH] OR "Depressive Disorder, Major" [MeSH] AND "Fatigue" 
[MeSH] OR "Fatigue Syndrome, Chronic" [MeSH] OR "Somatization disorder" [MeSH]. Papers published in English language until December 2018 were included. The inspection of the reference lists of the included studies and tracking citations of included papers in Google Scholar augmented this search strategy. Observational, experimental studies in human and animal models and relevant literature reviews were included. The overall methodological quality of retrieved references was considered for final inclusion.

\section{Microglia physiology overview: the good and the bad}

\subsection{Microglia ontogeny}

As firstly proposed by Rio-Hortega in 1919, different from other CNS cells that arise from the neuro-ectoderm, microglial cells are derived from the mesodermal cell lineage during embryogenesis [25]. Even when compared to mesoderm-originating myeloid cell, microglia differentiate from other monocytic cells. In fact, monocytic and macrophage-resident cells emerge from two waves of erythromyeloid pro-genitor (EMP) production (primitive and transient definitive hematopoiesis) in the extra-embryonic yolk sac (YS) before the adult bone marrow assumes the definitive hematopoiesis. Microglia, in turn, derives from the first hematopoietic wave at embryonic day (E) 7.5 (in mice). These primitive cells spread via the bloodstream to the neuroepithelium, which gets colonized by primitive macrophages (microglia) as early as E9.5. By contrast, other tissue-resident macrophages originate from the late EMP wave arising after the E8.5 [26]. Nevertheless, the functional consequences of those ontogeny differences between microglia and other resident macrophages are not clear. In fact, gene expression profiling studies demonstrate that microglia express several genes typical from myeloid lineage, such as receptors for pathogen-associated molecular patterns (PAMPs) and damageassociated molecular patterns (DAMPs), making their distinction from other macrophage cell sometimes difficult, especially under pathological conditions [27]. After the first wave of microglial ancestors, these primitive myeloid progenitors are in the essence the first glial cells in 
the CNS (as both astrogliogenesis and oligodendrogliogenesis occur later in a perinatal period). This coincides with the first phase of embryonic synaptogenesis around E14. Here, microglia assist, refine and even promote early synaptogenesis through secretion of growth factors, when astrocytes are absent [28]

\subsection{Microglia CNS morphology and functions}

In mammalians, the density of microglial cells increases progressively during the development of the CNS, ranging from 5 to $15 \%$ of the total cells in adult brain [29]. Throughout life, microglial cells maintenance and local expansion are almost exclusively dependent on selfrenewal of CNS-resident microglia, whereas progenitor recruitment from the circulation occurs only in special conditions. After migration to neuroepithelium, the precursor myeloid cells suffer remarkable changes to achieve its specific morphology and related functions (a small cell body endowed with several extra-thin and highly motile processes), which was termed "resting microglia". Resting microglia integrate the neural circuits and assumes important functions to CNS development, remodeling and plasticity [1]. However, increasing evidence has suggested that microglia show a great morphological heterogeneity between brain regions, which has been speculated to correspond to different physiological roles, which await further investigation [for detailed revision see [30]].

The classical paradigm of microglia as "resting cells" has been intensively questioned in the last years. A striking advance to this theory came from findings that showed microglial process motility in the living mouse by in vivo two-photon laser-scanning microscopy [28]. In these experiments, through a cranial window, microglial cells were observed in undisturbed CNS tissue, revealing that microglial processes show continuous fast movement at the order of 1 to $3 \mathrm{~mm}$ of extensions and retractions per minute [31,32].

Subsequent evidence showed that neuronal products or metabolites can control microglial movements. In this context, "resting" microglia morphology and process motility are 
tightly controlled by neurotransmission. They showed that ionotropic glutamatergic neurotransmission increased microglia motility, whereas ionotropic GABAergic neurotransmission reduced it. This effect was mediated indirectly via extracellular ATP concentrations, released in response to glutamatergic neurotransmission through probenecidsensitive pannexin hemichannels [33]. Additionally, lowering extracellular ATP concentrations results in reduction of microglia movements, and artificially creates ATP gradients progressively stimulating their motility [34]. ATP concentrations control microglial process movements through metabotropic P2Y12 purinoceptors expressed in their outer membranes [35].

Under conditions of disturbed brain homeostasis, including infection, injury, neurodegeneration, or markedly altered neuronal activity, microglial structure and function are rapidly and profoundly altered, assuming an 'activated' status characterized by (i) rapid and targeted movements of microglial processes toward the site of infection or injury; (ii) proliferation and resultant increase in the density of microglial cells; (iii) morphological alterations, including enlargement of the soma, increase in the diameter of primary processes, shortening of distal processes, and, in fully activated microglia, complete retraction of all processes and assumption of an amoeboid morphology; (iv) enhanced phagocytic activity; and (v) production and secretion of inflammatory cytokines and other mediators $[36,37]$. It is now evident that microglial activation is not an 'all or none' process, in other words, microglia can undergo multiple functional alterations or programs, which confer specific adaptation for coping with diverse pathological conditions [28].

The process of microglial activation is controlled by exogenous and endogenous 'alarm' molecules ('on' signals) or by suppressed production of microglia-inhibitory molecules that are constitutively produced in the brain, usually by neurons ('off' signals). Microglial activation and associated neuroinflammation evolved as an adaptive process, allowing the elimination of pathogenic challenges [1]; however, under many circumstances, activated microglial can acquire neurodegenerative roles, abnormally secreting toxic and alarm substances, such as 
reactive oxygen species, cytokines and catabolites, as well as by a physical contact with neurons these cell may eliminate functional synapses [38] (Figure 1).
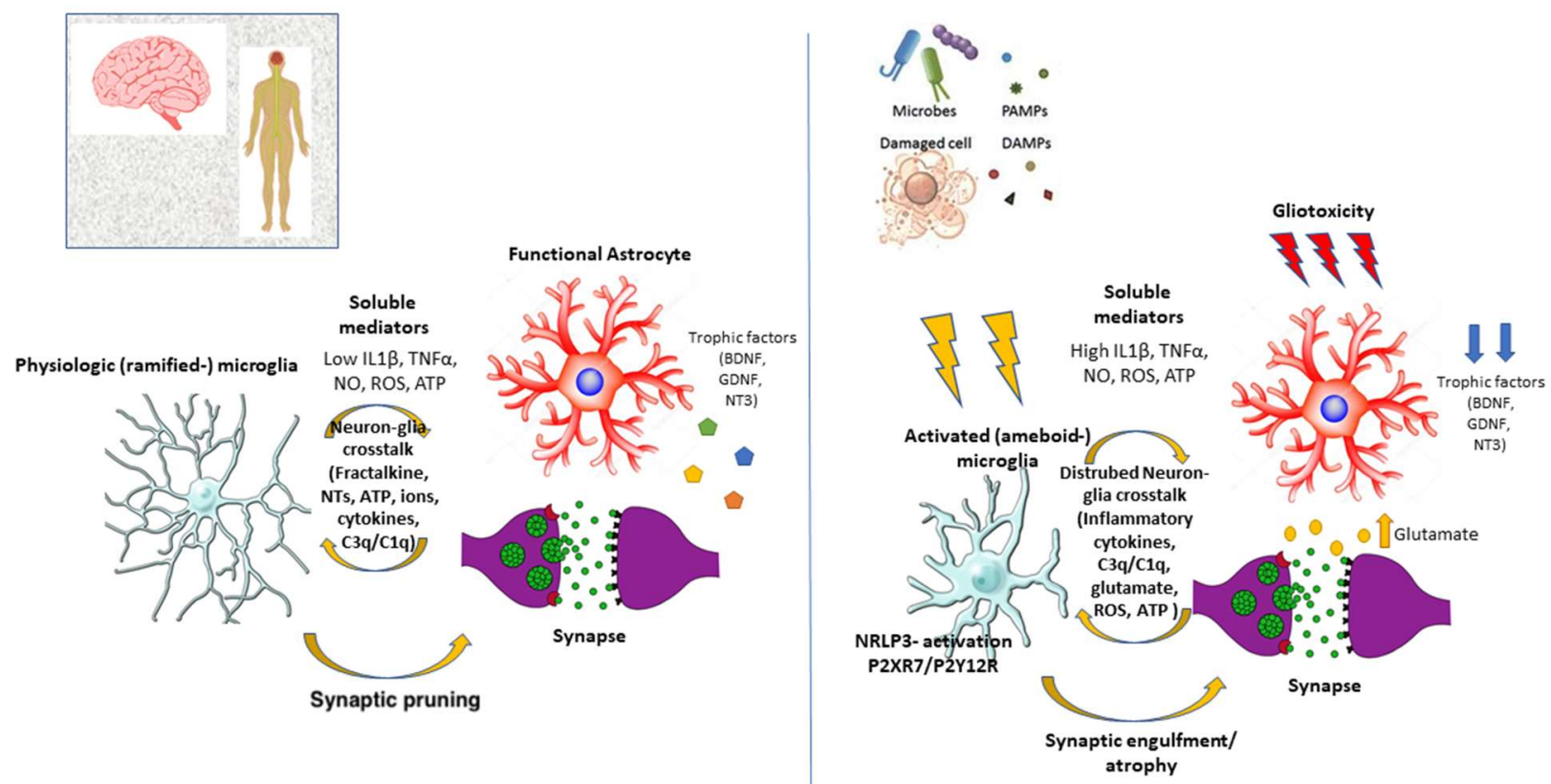

Figure 1. Microglia functions and phenotypes according to environmental stimuli. Resident microglia actively survey their own parcel of brain parenchyma and are referred to as surveying ramified microglia. Healthy neurons produce calming signals, such as fractalkine, that prevent inappropriate microglial activation, whereas microglia exert a trophic function via release of trophic factors, such as brain-derived neurotrophic factor (BDNF). Microglial processes have dynamic structural interactions with dendritic spines that physiologically regulate synaptic plasticity and pruning. Microglia respond to several types of CNS injury, codified through DAMPs and PAMPs, switching state to various types of activated (usually ameboid) phenotypes. In this activated state, microglia overproduce proinflammatory cytokines, such as IL-1 $\beta$ and TNF- $\alpha$, and cytotoxic substances such as NO and reactive oxygen species (ROS). Microglia-neuron and -astrocyte communication is also disturbed, for example, through increase in complement $(\mathrm{C} 1 \mathrm{q} / \mathrm{C} 3 \mathrm{q})$ and ATP released from astrocytes, accentuating the proinflammatory milieu and glutamate uptake deficit by astrocytes. Also, trophic factors production by microglia is usually impaired, also impacting the trophic support to neurogenesis and gliogenesis. CNS: central nervous system; IL-1ß: interleukin-1beta; TNF-a: tumor necrosis factor alpha; NO: nitric oxide; C1q/C3q: complement component; NTs: neurotransmitters; ROS: reactive oxygen species; DAMPs: damageassociated molecular patterns; PAMPs: pathogen-associated molecular patterns; BDNF: brain-derived neurotrophic factor; GDNF: : glia-derived neurotrophic factor; NT3: neurotrophin-3; NRLP3: inflammasome signaling pathway; ATP: adenosine triphosphate; P2Y12R/P2XR7: purinergic receptors. 


\subsection{Neuron-microglia interactions}

In the absence of insults, the dynamic surveillant microglia is specifically close to synaptic structures. Microglial processes situate near to presynaptic boutons, where they remain about $5 \mathrm{~min}$ and then retract [39]. These dynamic neuron-microglia interactions are activity-dependent, and this contact frequency reduces as neuronal firing diminishes. This phenomenon was particularly demonstrated in sensory experience. For this end, it was demonstrated that light deprivation reduced microglial processes motility and increased their association just with larger dendrites of retinal neurons. Re-exposure to light reversed this atrophy process and microglia ramifications contact more synapses [40].

Microglia regulate neuronal function and morphology through contact-dependent and independent mechanisms. Contact-dependent mechanisms include engulfment and phagocytoses of synaptic and dendritic elements [41]. Important studies demonstrated the striking relevance of microglia phagocytose of synaptic elements during neurodevelopment, a process called synaptic pruning [40]. In adult brain, microglia perform regular selection of adult synapses, called "synapse sampling". As abovementioned, microglia perform dynamic contacts with proximal synapses, mainly most active synapses. Formative studies showed that the prolonged contact with microglia results in synapse engulfment and loss [41]. For instance, abnormal glutamate release rapidly draws microglia processes toward these sites, which subsequently surrounding hyperactive neurons and leading to contact-dependent reductions in neuronal activity [42].

Additionally, microglia affect neural circuits through secretion of several bioactive factors, such as neurotransmitter, metabolites and neurotrophins (a contact-independent mechanism). These agents include, but are not restricted to: several types of cytokines such as tumor necrosis factor (TNF)- $\alpha$; chemokines such as CCR1, 3, 5, 7 and CXCR1; trophic factors like brain derived neurotrophic factor (BDNF); the gaseous transmitter nitric oxide (NO) or neurotransmitters (ATP and glutamate), and metabolites, such as tryptophan catabolites 
(kynurenine and quinolinic acid) [for review, see [43]]. These neuroactive substances can rapidly modulate neuronal functions, influencing membrane excitability and synaptic strength, through gene expression-dependent mechanisms. Despite their involvement in pathological processes, they can mediate interactions of microglia with synapses in the healthy brain. For example, fractalkine and complement factors regulate the synaptic pruning during development, while TNF- $\alpha$ has important participation in synapse sampling in adult brain $[44,45]$.

Notably, pro-inflammatory cytokines dependent effects are the best demonstrated mechanisms by which microglia control neuronal networks. For example, TNF- $\alpha$ is a proinflammatory cytokine, which is exclusively released by microglial cells and stimulate the production of other cytokines such as IL-1 $\beta$ and IL-6 [46]. Under physiological levels (about 100 picomolar concentrations), TNF- $\alpha$ is a potent effector of synaptic sampling, promoting a regular adjustment of the synaptic strength on a given neuron in response to prolonged electrical activity. At physiological concentrations, TNF- $\alpha$ can increase the ratio of AMPAR- to NMDARmediated synaptic currents without affecting long-term potentiation (LTP) or long-term depression (LTD) [46]. The TNF- $\alpha$ synaptic effect is mediated by its action on the astroglial TNF receptor I (TNFR I). TNF- $\alpha$, together with microglial release of ATP, causes an amplified ATP signal on adjacent astrocytes. This mechanism activates astroglial P2Y1 receptors triggering glutamate exocytosis [47]. In the hippocampus, increased astroglial glutamate release stimulates neurotransmitter exocytosis on presynaptic neurons. The electrical consequence of this phenomenon is the rinse in the excitatory postsynaptic current frequency of selected neurons [46]. Taken together, through contact-dependent and through soluble factors, microglia actively participate in homeostatic control of neural networks and synaptic activity (Figure 1).

\subsection{Microglia and CNS IL-1ß: close friends in health and disease}

Disturbances in microglial morphology/function are one of the most common neuropathological findings in several neuropsychiatric disorders. After acute injury, the 
surveillant spider-shaped ramified microglia assume an ameboid deramified phenotype described as activated state. Compelling evidence has reported the involvement of microglial activation in neurodegenerative and autoimmune CNS disorders, and as previously mentioned in this text, in some psychiatric disorders including depression $[3,36]$.

Activated microglia could differentiate into multiple states. Usually, the most inflammatory type is designated as M1 and the most anti-inflammatory type is designated as M2, despite there are some controversial opinions regarding microglia polarization into M1 and M2 phenotypes [37]. According to the polarization theory, M1 microglia express inflammatory cytokines including IL-1 $\beta$, TNF- $\alpha$, and iNOS, whereas M2 microglia express TGF- $\beta$, IL-4 or IL10, and arginase 1. Microglia are also the main, but not the only, source of IL-1 $\beta$ production in the brain in the absence of infiltrated leukocytes. Low concentrations of IL-1 $\beta$ stimulate noninflammatory actions in neuronal and non-neuronal cells, such as synaptic pruning and excitatory transmission, however, at high levels (as produced by microglia), IL-1 $1 \beta$ mounts the immunological changes that characterize CNS inflammation with increased cytokine production, reactive oxygen radical overexpression and neuronal damage [48].

The IL-1 family comprises 11 members, but two members are more prominent described in the literature, IL-1alpha (IL-1 $\alpha)$ and IL-1 $\beta$. Even though both cytokines bind and activate type 1 IL-1 receptor (IL- 1R1), IL-1 $\beta$ has been more associated to the inflammatory milieu. In fact, IL$1 \beta$ is synthetized by the subset of monocytic-like cells, including monocytes, macrophages, dendritic cells and CNS microglia. This cytokine is produced as a precursor and in order to become biologically active needs to be cleaved by caspase-1, an intracellular enzyme that is activated by a complex of intracellular proteins termed "the inflammasome" [49]. IL-1 can also be cleaved by an alternative mechanism dependent of neutrophil enzymes, such as elastase and proteinase-3. After binding to its receptor, IL-1 $\beta$ induces the activation of a complex cascade that culminates in activation of transcriptional factors, such as "nuclear factor kappalight-chain-enhancer of activated B cells" (NFKB), and subsequent gene expression [50]. 
IL-1 $\beta$ presents important CNS effects, and most of them were investigated in the context of immune activation with LPS, and the emergence of sickness-behavior. Hence, the following brain functions are regulated by $\mathrm{IL}-1 \beta$ : i) temperature in the preoptic-anterior hypothalamus being one mediator of fever [51,52]; ii) sleep cycle, since increased expression of this cytokine induces spontaneous sleep, a dose-dependent increase in non-rapid eye movement (non-REM) sleep, as well as suppression of wakefulness and REM sleep [53-55]; iii) neurotransmission by mechanisms such as inhibition of $\mathrm{Ca}+$ channel currents, reduction of GABA A receptor- and NMDA receptor- mediated responses, potentiation of non-selective cationic conductance and activation of voltage-dependent sodium currents as well as voltagedependent potassium currents [56]; iv) memory formation based on findings that at low noninflammatory concentrations, IL-1 $\beta$ may facilitate long-term potentiation (LTP) and synaptic strength, whereas at high inflammatory levels, this cytokine consistently impairs LTP and memory process $[57,58]$; v) trophic effects in microglia by a purinergic action in microglial activation and proliferation under injury conditions [59]; vi) neurogenesis, since IL-1 $\beta$ mediates the inhibitory effects of interferon- $\mathrm{\gamma}$ (IFN- $\mathrm{\gamma}$ ) treatment and chronic stress protocols in the rate of neurogenesis in adult mice hippocampus [60]; vii) neuroendocrine functions based on the findings that IL-1 receptor knockout or transgenic mice overexpressing brain IL-1ra present reduced $\mathrm{ACTH}$ and glucocorticoid responses in psychological and metabolic stress models, while intracerebral administration of IL-1 $\beta$ induces the secretion of glucocorticoid hormones $[61,62]$.

It is worth mentioning that this regulation of metabolism by IL-1 $1 \beta$ occurs despite of hypoglycemia [56]. In this regard, neuronal IL-1 synthesis is induced by stimulation of AMPA receptors on neurons and the resulting release of $\mathrm{IL}-1 \beta$ can stimulate glucose uptake by neurons in an autocrine or paracrine fashion [63]. Overall, it is interesting to speculate that these physiological activities of IL-1 might coordinate with its immune activities such that hyper- 
inflammation may be prevented, and brain energy usage may be spared even when immune activity might be energetically costly [56].

Other cytokines, such as TNF- $\alpha$ and IL-6, also have several modulatory actions under neurotransmission, circadian rhythms, glucocorticoid responses and neurogenesis [for detailed review, see [64]].

\section{Microglial disturbances in depression - depression as a microgliopathy}

Animal models provided valuable evidence for the role of microglia in depression. The evidence came from studies evaluating microglia responses to immune challenges such as LPS (for detailed review, see [65]). On the other hand, depressed patients present neuroinflammatory alterations, probably related to microglia activation [38], while recent evidence point towards increased LPS levels in the plasma of depressed patients $[14,66]$.

\subsection{Preclinical findings based on immune challenge}

LPS is an endotoxin from gram-negative bacteria whose systemic administration in rodents induces time-dependent behavioral alterations that resemble depression, being thus considered a useful inflammation-based model of depression. In this model, several findings indicate the contribution of activated microglia to the onset of depressive-like behaviors [65]. Indeed, $24 \mathrm{~h}$ after a single LPS administration, a time point referred as depression, there is an increased expression of markers of microglial activation in limbic brain areas. These changes are followed by increased levels of pro-inflammatory cytokines, namely IL-1 $\beta$, IL-6, and TNF- $\alpha$, oxidative damage and impairment of hippocampal neurogenesis [67-69].

Also, mice with microglial hyper-reactivity, induced by a microglia-fractalkine receptor mutation [70] or by traumatic brain injury [71], exhibit exacerbated LPS-induced depressive-like symptoms. The tetracyclines, minocycline and doxycycline, known for their microglial inhibition 
properties, have the ability to counteract both behavioral and neuroinflammatory changes induced by LPS [71,72].

Recently, brain region- and dose-dependent effects of LPS challenge were described in microglia populations. In line with this, low doses of LPS (at $100 \mu \mathrm{g} / \mathrm{kg}$ ) can induce robust microglial proliferation in circumventricular organs (CVOs) and their neighboring brain regions. In turn, immune stimulation with higher LPS doses $(1 \mathrm{mg} / \mathrm{kg}$ ) resulted in a rapid increase in microglia density and proliferation not only in CVO, but also in hypothalamus, medulla oblongata, and limbic system that was maintained for 3 weeks [73]. Recently, our research group showed that a repeated ( 7 days out of 30 days) and intermittent exposure (4 months) of mice to fluctuating doses of LPS ranging from 750 to $1250 \mu \mathrm{g} / \mathrm{kg}$, in each 7 days treatment interval, causes long-lasting depressive-like behavioral changes accompanied by hippocampal alterations. These hippocampal alterations included: i) increased immunoexpression of ionized calcium-binding adapter molecule 1 (IBA1), a microglia marker, ii) increased expression of NF-kB p65 and IL-1 $\beta$, iii) increased IDO1 mRNA expression, an enzyme present in microglial cells and related to tryptophan catabolism, being activated by pro-inflammatory cytokines [74].

\subsection{Pre-clinical findings based on stress models}

In stress-based models, microglia activation participates in the development of depressive-like changes. For instance, acute stressors, such as restraint stress and electric shock, induce microglial activation in many brain areas, but mainly in midbrain periaqueductal gray (PAG), an area that plays important roles in behavioral responses to threat, anxiety, and pain $[75,76]$. Interestingly, minocycline blocked the effects of foot-shock stress on hypothalamic IL-1 $\beta$ and microglia activation [77]. Semi-chronic (2-6 days) repeated restraint also induced a dramatic increase in the proliferation of hippocampal microglia together with increased gene expression and immunostaining of several markers of activated microglia [78]. 
Social defeat stress is an interesting model based on the exposition of intruder mice to aggressive resident partners. In this model, social stress not only induces activation of brain resident microglia, but also increases the infiltration of peripheral macrophages into the brain and their transformation in microglia-like cells $[79,80]$. These latter cells produce higher levels of IL-1 $\beta$ compared to resident microglia and show increased phagocytic activity [81]. Moreover, it was reported that mice previously exposed to social defeat stress develop a more robust inflammatory response and microglial activation to a subsequent immune challenge [79]. In fact, socially-defeated mice when injected LPS show an increased expression of IL-1 $\beta$, TNF- $\alpha$ and iNOS, together with increased density of $\mathrm{CD}_{11} \mathrm{~b}^{+}$cells (microglial marker) and CD11 $\mathrm{b}^{+} / \mathrm{CD} 45$ (high) cells, an index of bone marrow-derived microglia [82]. Therefore, social stress may act as a priming event for microglia making them more vulnerable to secondary immune-based events.

Chronic stress exposure also results in activation of brain microglia. Indeed, exposure to homotypic chronic stress (restraint stress) for 14 and 21 days resulted in increased microglial proliferation, hyper-ramification (i.e., branching) of processes, and expression of the microglia marker IBA1. These changes were followed by depressive-like and anxiety alterations [83-85]. Furthermore, exposure to chronic unpredictable stress (CUS) protocols also resulted in marked microglial activation. In line with this evidence, a recent study showed that the exposure to 12 weeks of CUS induced remarkable hippocampal microglial activation detected through several methodologies: TSPO-PET imaging, immunofluorescence staining and protein expression (western blotting). Of note, TSPO is a protein present in the outer membrane of mitochondria in central and peripheral tissues being robustly enhanced in reactive microglia and astrocytes. These animals also show increased markers of NLRP3 inflammasome activation and proinflammatory cytokines (IL-1 $\beta$, IL-6, and IL-18) in the hippocampus. Interestingly, minocycline treatment reversed both behavioral and neuroinflammatory changes [10]. In the context of neuroinflammation, exposure to CUS exacerbated microglial activation induced by intracerebral 
administration of LPS [85]. Importantly, the effects of chronic stress on microglial activation are heterogeneous and suffer influence of several parameters, including gender and age of the animals, the intensity of stressful events applied, their frequency and the duration of protocol [86].

Early-life stress adversity and trauma are major risk factors for the development of depression [87]. Early-life stress can hamper the immune functioning at long-term, contributing to abnormal endocrine, behavioral and neural response to subsequent stressful situations and increased risk for psychopathology [88]. Indeed, prenatal stress, which is known to promote subsequent depressive-like symptomatology in the offspring, was shown to induce in mice longlasting basal hippocampal microglial activation together with an exacerbated microglial responsiveness to systemic LPS administration [89]. Furthermore, adult mice that were exposed to neonatal maternal separation displayed increased number and motility of microglial processes $[90,91]$. Also, maternally separated mice showed increased microglial response to a second stressful event in adolescence (food deprivation), indicating that early-life adversity acts as a priming event for microglia reactivity, increasing the risk of an abnormal response to a second stimulatory event [92]. Further studies should be designed to confirm the possible causal correlation between early-life stress induced microglia activation and psychopathology outcomes in clinical samples.

\subsection{Clinical findings}

Various bacterial and viral infections (e.g., Gram-negative bacteria, influenza virus, Epstein-Barr virus, herpesvirus, cytomegalovirus, Borna disease virus, and gastroenteritisrelated viruses) are associated with a range of depressive symptoms [93,94]. Many of these pathogens induce not only a peripheral but also a central pro-inflammatory response, which correlates with the emergence of neuropsychiatric symptoms [95]. In a similar way as seen in rodents, systemic administration of LPS to healthy humans induces a wide-spectrum of 
neurobehavioral alterations with similarities to depression symptomatology. These behavioral alterations in humans were tightly correlated to blood levels of pro-inflammatory markers $[96,97]$, and more recently, it was reported that systemic LPS also induces a remarkable microglial activation, indicated by translocator protein $18 \mathrm{kDa}$ (TSPO)-PET binding in the living human brain. These authors also found that LPS caused a marked increase in peripheral inflammatory cytokine levels and self-reported sickness symptoms [98].

Regarding depressed patients, the evidences of microglia activation in these patients are controversial and still inconclusive. Specifically, in post-mortem studies, one study demonstrated the presence of activated microglia in one of six affective disorder patients [99], and sequential studies reported no differences between depressed patients and controls in microglial density [38]. In PET imaging studies this scenario is a little clearer. In 2013, Hannestad and coworkers did not find significant difference in total or specific brain region TSPO binding between depressed and control patients [100]. Setiawan and coworkers showed that TPSO binding in prefrontal cortex, anterior cingulate cortex, and insula keeps a strong correlation with total illness duration $(P=0.0021)$ and duration of antidepressant treatment $(P=0.037)$. Notably, in patients with untreated disorder for 10 years or longer, TSPO binding was $29-33 \%$ greater in the mentioned areas than in participants who have less duration of illness [101]. Additionally, Richards et al., 2018, corroborating the previous findings, showed an increased TSPO binding in subgenual prefrontal cortex (sgPFC) and anterior cingulate cortex (ACC) of untreated depressed patients compared to controls. Interestingly, these researchers found a significant correlation between the TSPO results and IL-5 in cerebrospinal fluid of patients [102]. One important finding that may explain microglial activation in depressed patients is the presence of increased levels of plasma LPS due to increased intestinal barrier permeability $[14,66]$.

Suicide may also be associated with microglial activation. In fact, in the prefrontal cortex of suicide patients (independently of psychiatric diagnoses), an increased density of activated 
microglia, as well as macrophage-derived microglia and microglia immunoreactive cells in contact or within blood vessel was found [103]. Similar findings were reported in the hippocampus [104] and dorsal anterior cingulate of suicide patients [105] (with depression or other psychiatric diagnose). Regarding TSPO PET imaging studies, a recent study corroborated the aforementioned post-mortem reports, showing that TSPO binding was robustly increased in the anterior cingulate cortex and insula of depressed patients with suicidal thoughts in relation to depressed patients without suicide thinking [106]. Despite the enthusiasm with the use of TSPO ligands as neuroimaging markers of microglial activation, these results should be interpreted with caution and some limitations should be considered [please, read [107] for more details].

\section{Microglia disturbances and cytokine-mediated immune activation as core mechanisms of fatigue}

\subsection{Central Inflammation as a mechanism for fatigue symptom in the course of diseases}

There is now also evidence that central inflammation and pro-inflammatory cytokines are essential factors for the development of fatigue in the course of psychiatric and non-psychiatric diseases $[108,109]$. In this context, after an immune stimulatory event (such as bacterial or viral infection), elevation of brain pro-inflammatory cytokines mediates the emergence of a cluster of behavioral alterations described as "sickness behavior". Sickness includes symptoms of fatigue, loss of social interest, depressed mood, reduction in physical energy and circadian disturbances. In the experimental setting, these alterations have been observed after systemic and intrathecal administration of pro-inflammatory cytokines, and consistent data pointed to the central role of IL-1 $\beta$ as inflammatory mediator of fatigue symptom.

It is important to emphasize that sickness behavior, depression and chronic fatigue are not the same entities [110]. Sickness behavior is an adaptive response that enhances recovery by conserving energy to combat acute inflammation, whereas depression and chronic fatigue are related to a sensitization of immuno-inflammatory pathways, progressive damage by 
oxidative and nitrosative stress to lipids, proteins, and DNA, and autoimmune responses directed against self-epitopes, being thus considered as neuroprogressive processes [111].

\subsection{Microglial activation and pro-inflammatory cytokine overproduction as biological pathways} underlying fatigue

Growing evidence has linked inflammatory cytokines and chronic inflammation to the suppression of neural drive of the circadian pacemaker. Circadian disruption represents one of most established pathological changes involved in fatigue. Indeed, in chronic fatigue, it is postulated that a muffled daily signal from the circadian clock lead to reduced energy arousal, impaired mental concentration and increased fatigability [112]. This loss of rhythmic signal also affects other brain areas potentially impairing central functions, such as motivation, appetite control and pain threshold [112]. The unbalance in circadian synchrony also occurs in peripheral organs and could bring general feelings of malaise like that experienced during jet lag or rotating shift work. Reduced wakefulness and locomotor activity also impair the feedback that locomotor activity normally exerts in the sculpting of daily rhythmic output, further damping rhythm output. Circadian disorganization also influences metabolism and immune function $[113,114]$.

The key controller of circadian rhythm is the suprachiasmatic nuclei (SCN) in the hypothalamus [115]. In humans and monkeys, SCN lesions produce a markedly reduction of total wakefulness time and induces fatigue symptoms. As a master controller, SCN send projections to important regions for regulating sleep/ wake cycle, locomotor arousal and reward. Fibers from SCN reach both the wake-promoting neurons and the sleep-promoting systems by direct and indirect routes, relayed through the dorsomedial hypothalamus (DMH) and the ventral subparaventricular zone [116]. The SCN also modulates the firing rate of noradrenergic neurons of Locus ceruleus through projections to orexin-positive neurons of DMH [117]. Finally, SCN communicates with a subpopulation of neurons of ventral tegmental area (VTA), controlling the 
diurnal activity of dopaminergic neurons and the circadian oscillation of reward-seeking behavior [118].

Cytokines can greatly affect SCN functioning. Indeed, receptors for IL-1 $\beta$, TNF- $\alpha$ and interferons are expressed in ventrolateral SCN of rodents, and immune challenges including LPS or pro-inflammatory cytokines result in marked changes in circadian rhythm and in synaptic activity of SCN [113]. For example, IFN- $\alpha$, the type I interferon widely used for its antiviral and antitumor agent properties is capable of inducing depressive symptoms, disrupt the ability of the biological clock to drive endogenous rhythms, as well as suppress clock gene expression in the SCN and periphery [119]. Gene expression in the SCN and its function also show timedependent changes after systemic LPS challenge [120] and these changes are dependent of TLR4 activation as previously demonstrated [121]. Additionally, Bentivoglio et al. (2006) performed intracerebroventricular (ICV) injections of pro-inflammatory cytokines and found an important glial reactivity in several hypothalamic nuclei, but mainly in the circadian pacemaker SCN [122]. These authors observed a robust increase in the density and immunoreactivity of astrocyte and microglia markers. Regarding microglial cells there are marked morphological changes: enlarged cell body and reduction of ramification processes, that characterizes as activated microglia. These researchers also noted that this glial response was exacerbated with aging, a condition that is accompanied by low-grade inflammation [122]. Similarly, activated microglia and reduced circadian amplitude and alterations in spontaneous activity levels were observed in mice infected with simian immunodeficiency virus, another condition characterized by chronic low-grade inflammation [123].

Important evidence of microglial activation in circadian abnormalities and fatigue came from studies on "sleeping sickness", a condition caused by the protozoan parasite Trypanosoma brucei (T. brucei). This parasite invades the CNS and induces several clinical manifestations, including sleep/wake disturbances, cognitive deficits and marked 
fatigability. In rodents, T. brucei infection also showed reduced amplitude of circadian rhythm, reduction in spontaneous activity and impairments in SCN spontaneous firing [124]. Previous research reported that this protozoan causes marked neuronal degeneration, astrocyte reactivity [125] and glutamate receptor disturbances in the SCN [124].

Marked changes have also been observed in microglia of T. brucei infected mice. Firstly, Chianella and coworkers showed a remarkable activation of hypothalamic microglia that follows a progressive weekly course and parallels progressive sleep and activity alterations until terminal stages [126]. Recently, Figarella et al. through an ultrastructural analysis confirmed the ability to $T$. brucei to induce microglial activation [127].

\subsubsection{Neuroinflammation in animal models of fatigue}

There are some animal models based on neuroinflammatory alterations in fatigue. The first one is called central fatigue model (CFM) and is based on the cortical spreading depression (SD), that is, the propagation of neuronal membrane depolarization throughout the cerebral cortex. In CFM there is an increased expression of COX-2 in cortical neurons with the resulting production of prostaglandins (PG) including PGD2 and increased amount of non-REM sleep, but not of REM sleep. Of note, a COX-2 inhibitor blocked the increase in non-REM sleep.

The second model, the immunological fatigue model, is based on the systemic administration of polyriboinosinic:polyribocytidylic acid (poly-I:C) to rodents. Poly I:C is a viral mimetic particle that causes a sustained reduction in spontaneous activity and well-being in rodents for weeks, when sickness-behavior and acute-phase responses already ends, being considered as an useful model of inflammation-induced fatigue [128]. Intraperitoneal poly I:C administration induces marked microglial activation and IL-1 $\beta$ in mice prefrontal cortex. This IL$1 \beta$ up-regulating effect was also observed in microglia cultures exposed to poly I:C [129]. The importance of IL-1 $\beta$ for poly I:C-induced fatigue symptoms was demonstrated recently, once 
ICV administration of IL-1 $\beta$ neutralizing antibody [129] or of a IL-1 receptor antagonist [130] prevented microglial activation and fatigue-related symptoms. Similarly, minocycline treatment prevented IL-1 $1 \beta$ rise and induction of fatigue. These researchers also investigated the participation of other cytokines, such as IFN- $\alpha$, in this effect and concluded that poly I:C-induced fatigue depends on IL-1 $\beta$ synthesis [129].

All in all, there is evidence that under conditions of chronic or abnormal inflammation, such as infections and senescence, glial cells, mainly microglia, orchestrate an inflammatory microenvironment in pacemaker circuits responsible for the control of circadian and energy arousal which may cause symptoms of fatigue.

5.4 Evidence of microglial activation and pro-inflammatory cytokine disturbances in Chronic Fatigue Syndrome (CFS)

Chronic Fatigue Syndrome is characterized by severe and prolonged fatigue as well as by marked circadian abnormalities [131]. The pathophysiology of CFS is not well understood, and as other chronic diseases, several pathways are probably involved. In this context, some recent studies have reported that neuroinflammation can be an important underlying factor for this disorder. In this context, direct evidence of microglial disturbances in CFS patient population is very scarce. Just a single study tested TSPO binding through PET imaging study in CFS patients and found an increased widespread brain signal for this marker, an promising indicative of microglial activation [132]. Other imaging studies showed disturbed connectivity in prefrontal cortex and frontal networks in these patients $(19,20)$, as well as impaired glutamate levels in this brain area $[133,134]$. On the other hand, regarding cytokines, several investigators have measured these markers levels in the blood of CFS patients, and interesting associations between IL-1 and other cytokine levels and fatigue symptoms have been reported [24].

In this context, a consistent systematic review was conducted by Blundell and coworkers evaluating cytokine serum levels abnormalities in CFS patients. These researchers 
revised 38 studies between 1988 and 2015, including the first case report of CFS in the literature. These authors found 28 studies measuring IL-1 $\beta$ levels, $25 \%$ of them reporting increased levels of this cytokine in the plasma of CFS patients compared to controls, whereas 11 studies evaluated IL-1a levels with $27 \%$ of them observing increased levels of this cytokine. Blundell et al. also reported increased levels of transforming growth factor-beta (TGF- $\beta$ ) in CFS patients [135]. After Blundell's review, three new studies evaluating serum cytokines in CFS patients were published. Russel et al., found no differences in IL-1 levels between patient subgroups, although IL-1 $\alpha$ appeared to have predictive value in recently ill adolescent patients [136]. Hardcastle et al. found increased IL-1 $\beta$ levels in the serum of moderately ill patients compared to controls [137], and the third study could not find any differences between patients and controls for either IL-1 $\beta$ or IL-1 $\alpha$ in a group of 100 patients and 79 controls [138].

Another approach in this study is to compare cytokine production of peripheral blood mononuclear cells (PBMCs) after immune stimulation. An early study described increased IL-1 $\beta$ production after LPS challenge to PBMC of CFS patients compared to controls [139]. However, subsequent studies did not confirm this finding, observing lower IL-1 $\beta$ response after an immune challenge in CFS subjects or absence of differences between patients and controls $[140,141]$. IL-1 $\beta$ production by PBMCs has also been investigated in infection-related fatigue syndrome. It was reported that during the acute phase of infection, IL-1 $\beta$ concentrations correlates positively with fatigue symptoms, but this association was no longer present in the persistent phase of the syndrome $[142,143]$.

Since the CNS may be a key site underlying prolonged fatigue symptoms, studies have also evaluated cytokine levels in the cerebrospinal fluid. While two studies found no significant differences in cytokine levels in cerebrospinal fluid between patients and controls [144,145], another study reported lower IL-1 $\beta$ and IL-1Ra concentrations in the cerebrospinal fluid of CFS patients compared to multiple sclerosis and control groups [146], pointing to abnormalities in this cytokine signaling in the CNS [24]. 
Besides the involvement of IL-1 in fatigue symptoms and chronic fatigue syndrome, also other cytokines may play a role in CFS. Montoya and coworkers evaluated the serum of 192 CFS patients and 392 controls using a cytokine multiplex system and traced correlations with fatigue symptoms. They found that 17 cytokines positively correlated with fatigue symptom severity: CCL11 (Eotaxin-1), CXCL1 (GROa), CXCL10 (IP-10), IFN-y, IL-4, IL-5, IL-7, IL-12p70, IL-13, IL-17F, leptin, G-CSF, GM-CSF, LIF, NGF, SCF, and TGF- $\alpha$. Of them, thirteen are proinflammatory, confirming the strong involvement of immune activation in this disorder [147]. In accordance, another recent study found correlations between worse sleep quality and higher blood levels of IL-1 $\beta$, IL-6 and TNF- $\alpha$ in CFS. Sleep symptoms, in turn, predicted more severe fatiguability and impairment in daily activities in these patients [148].

Additionally, cell-mediated immune responses and autoimmunity have also been highlighted in CFS biology. In fact, Maes et al., 2012 reported increased levels of proinflammatory cytokines (IL-1 $\beta$ and TNF- $\alpha$ ), PMN-elastase and lysozyme and cell-mediated immunity markers (namely neopterin) in CFS serum. Interestingly, these cell immunity markers were significantly correlated with severity of fatigue symptoms in these patients [149]. Also, Maes et al., 2013 reported that increased inflammatory markers were accompanied by increased autoimmune responses against 5- hydroxytryptamine $(5-\mathrm{HT})$, as demonstrated by increased auto-antibodies levels in the serum of these patients [150]. Other studies also reported the high incidence of auto-antibodies to 5-HT and other neural epitopes, such as gangliosides and phospholipids, in patients with CFS and their relatives [151-153].

5.5 Involvement of pro-inflammatory cytokine overproduction and possibly microglia activation in depression and chronic fatigue and their comorbidity

Despite the evidence of the involvement of microglial disturbances and pro-inflammatory activation in the neurobiology of depression and depression symptomatology, not many studies 
examined the associations between immune-inflammatory markers and fatigue and physiosomatic symptoms in depression.

In this context, Jokela et al., 2016 provides a consistent evidence for the link between systemic inflammation and fatigue/somatic symptoms in depression. These researchers collected data of specific symptoms of depression and serum C-reactive protein from 3 crosssectional studies of more than 15,000 participants. They found an independent association of Creactive protein levels with physio-somatic symptoms of tiredness, fatigue, sleep problems and changes in appetite [154]. Additionally, Maes et al., 2012 showed increased levels of proinflammatory cytokines, namely TNF- $\alpha$ and IL-1 and neopterin in the serum of depressed patients, as well as observed that these pro-inflammatory changes were predominantly associated with the presence of fatigue and physio-somatic symptoms [149]. Recently, a longitudinal population-representative birth cohort study reported that IL-6 and C-reactive protein serum levels at childhood predict somatic/neurovegetative symptoms (including fatigue) in adult depressed patients [155]. Somatic symptoms prevalence and severity in depression were also associated with increased serum levels of sIL-2R and sP-selectin, a marker of vascular inflammatory dysfunction $[156,157]$.

Additionally, in woman with perinatal depression, the physio-somatic symptoms of pregnancy (fatigue, back pain, muscle pain, dyspepsia, obstipation) correlated positively with serum acute-phase proteins (C-reactive protein and haptoglobin) and $\operatorname{lgM}$ responses to the neurotoxic tryptophan catabolites (TRYCAT), such as quinolinic acid, whereas they were inversely correlated with serum tryptophan (TRP) levels [158]. In line with these sex-specific effects, Dannehl et al., 2014 reported that a history of fatigue and somatoform symptoms represents an important predictor of prospective high levels of TNF- $\alpha$ in the serum of women with major depression [159].

Interestingly, the reciprocal relationship between both depression and CF is also observed in patients with CFS in whom depressive symptoms are correlated with pro- 
inflammatory markers. Raison et al. showed that depressive symptoms in CFS patients correlates directly with serum high-sensitive C-reactive protein and white blood cell count (WBC) levels [160]. Also, it was reported that plasma levels of TNF- $\alpha$ and IL-10 associate positively with depression score at The Hospital Anxiety and Depression Scale (HADS) and SCL-90-R cluster somatization in CFS patients and controls [161]. Additionally, Milrad and coworkers using a structural equation modeling showed that depressive symptoms severity predicts higher serum pro-inflammatory cytokines (IL-2, IL-6 and TNF- $\alpha$ ) and elevated cortisol levels at evening in CFS female patients [162]. Further, regarding TRYCATs pathway, Maes et al., 2011 showed that severity of somatization more than other depressive symptoms positively correlates with the increase in neurotoxic TRYCATs in serum as indicated by the kynurenine/ tryptophan (KY/TRP) and kynurenine/kynurenic acid (KY/KA) ratios, and correlates negatively with serum levels of tryptophan [163].

Despite the considerable evidence for the association between microglial activation (through TSPO binding) with time of illness and severity in depression [10], as well as with the increased signal of this marker in the brain of CFS patients [132], to date, no study demonstrated the association of fatigue and somatic symptoms of depression with markers of microglial activation in living human brain. Future research should prioritize the examination of microglial activation in comorbid depression and CFS.

\section{Conclusion and future remarks}

In summary, current evidence indicates that an unbalanced immune response with a systemic pro-inflammatory state is an important underlying biological mechanism explaining the development of fatigue and other physio-somatic symptoms in depression. Likewise, in CFS, immune activation is associated with increased severity of depression and depressive symptoms, while shared immune pathways may also underpin the comorbidity between depression and CFS [164]. Based on our review we may posit that shared immune- 
inflammatory pathways and activated microglia underpin comorbid depression and CFS and that activated microglia is the main orchestrator of this comorbidity. As such, microglial activation and neuro-inflammation may be promising targets to treat the overlapping manifestations of both depression and CFS.

\section{References}

[1] J.A. Kabba, Y. Xu, H. Christian, W. Ruan, K. Chenai, Y. Xiang, L. Zhang, J.M. Saavedra, T. Pang, Microglia: Housekeeper of the Central Nervous System, Cell. Mol. Neurobiol. 38 (2018) 53-71. doi:10.1007/s10571-017-0504-2.

[2] D. NAYAK, T.L. ROTH, D.B. MCGAVERN, Microglia development and function, Annu. Rev. Immunol. 32 (2014) 367-402. doi:10.1146/annurev-immunol-032713-120240.

[3] A.R. Santiago, L. Bernardino, M. Agudo-Barriuso, J. Gonçalves, Microglia in Health and Disease: A Double-Edged Sword, Mediators Inflamm. 2017 (2017) 7034143. doi:10.1155/2017/7034143.

[4] Y. Wu, L. Dissing-Olesen, B.A. MacVicar, B. Stevens, Microglia: Dynamic Mediators of Synapse Development and Plasticity, Trends Immunol. 36 (2015) 605-613. doi:10.1016/j.it.2015.08.008.

[5] V.H. Perry, J.A.R. Nicoll, C. Holmes, Microglia in neurodegenerative disease, Nat. Rev. Neurol. 6 (2010) 193. https://doi.org/10.1038/nrneurol.2010.17.

[6] D. Brites, A. Fernandes, Neuroinflammation and Depression: Microglia Activation, Extracellular Microvesicles and microRNA Dysregulation, Front. Cell. Neurosci. 9 (2015). doi:10.3389/fncel.2015.00476.

[7] G.Z. Réus, G.R. Fries, L. Stertz, M. Badawy, I.C. Passos, T. Barichello, F. Kapczinski, J. Quevedo, The role of inflammation and microglial activation in the pathophysiology of psychiatric disorders, Neuroscience. 300 (2015) 141-154.

doi:10.1016/j.neuroscience.2015.05.018. 
[8] A. Monji, T. Kato, S. Kanba, Cytokines and schizophrenia: Microglia hypothesis of schizophrenia., Psychiatry Clin. Neurosci. 63 (2009) 257-65.

[9] E.S. Wohleb, Neuron-Microglia Interactions in Mental Health Disorders: "For Better, and For Worse," Front. Immunol. 7 (2016) 544. doi:10.3389/fimmu.2016.00544.

[10] Y.-L. Wang, Q.-Q. Han, W.-Q. Gong, D.-H. Pan, L.-Z. Wang, W. Hu, M. Yang, B. Li, J. Yu, Q. Liu, Microglial activation mediates chronic mild stress-induced depressive- and anxiety-like behavior in adult rats., J. Neuroinflammation. 15 (2018) 21. doi:10.1186/s12974-018-1054-3.

[11] R. Yirmiya, Endotoxin produces a depressive-like episode in rats, Brain Res. 711 (1996) 163-174. doi:10.1016/0006-8993(95)01415-2.

[12] M. Maes, W. Stevens, L. DeClerck, C. Bridts, D. Peeters, C. Schotte, P. Cosyns, Immune disorders in depression: higher T helper/T suppressor-cytotoxic cell ratio., Acta Psychiatr. Scand. 86 (1992) 423-31.

[13] C.S. Custodio, B.S. Mello, R.C. Cordeiro, F.Y. de Araujo, J.H. Chaves, S.M. Vasconcelos, H. V Nobre Junior, F.C. de Sousa, M.L. Vale, A.F. Carvalho, D.S. Macedo, Time course of the effects of lipopolysaccharide on prepulse inhibition and brain nitrite content in mice, Eur J Pharmacol. 713 (2013) 31-38. doi:10.1016/j.ejphar.2013.04.040.

[14] B.R. Stevens, R. Goel, K. Seungbum, E.M. Richards, R.C. Holbert, C.J. Pepine, M.K. Raizada, Increased human intestinal barrier permeability plasma biomarkers zonulin and FABP2 correlated with plasma LPS and altered gut microbiome in anxiety or depression, Gut. (2017) gutjnl-2017-314759. doi:10.1136/gutjnl-2017-314759.

[15] M. Maes, M. Kubera, J.C. Leunis, The gut-brain barrier in major depression: intestinal mucosal dysfunction with an increased translocation of LPS from gram negative enterobacteria (leaky gut) plays a role in the inflammatory pathophysiology of depression., Neuro Endocrinol. Lett. 29 (2008) 117-124. doi:NEL290108A12 [pii].

[16] M. Maes, M. Kubera, J.C. Leunis, M. Berk, M. Geffard, E. Bosmans, In depression, 
bacterial translocation may drive inflammatory responses, oxidative and nitrosative stress (O\&NS), and autoimmune responses directed against O\&NS-damaged neoepitopes, Acta Psychiatr. Scand. 127 (2013) 344-354. doi:10.1111/j.1600-0447.2012.01908.x.

[17] S.-A. Jeon, E. Lee, I. Hwang, B. Han, S. Park, S. Son, J. Yang, S. Hong, C. H. Kim, J. Son, J.-W. Yu, NLRP3 Inflammasome Contributes to Lipopolysaccharide-induced Depressive-Like Behaviors via Indoleamine 2,3-dioxygenase Induction, Int. J. Neuropsychopharmacol. 20 (2017) 896-906. doi:10.1093/ijnp/pyx065.

[18] S.P. Leighton, L. Nerurkar, R. Krishnadas, C. Johnman, G.J. Graham, J. Cavanagh, Chemokines in depression in health and in inflammatory illness: a systematic review and meta-analysis, Mol. Psychiatry. 23 (2017) 48. https://doi.org/10.1038/mp.2017.205.

[19] M. Maes, Inflammatory and oxidative and nitrosative stress pathways underpinning chronic fatigue, somatization and psychosomatic symptoms, Curr. Opin. Psychiatry. (2009). doi:10.1097/YCO.0b013e32831a4728.

[20] M. Maes, F.N.M. Twisk, M. Kubera, K. Ringel, Evidence for inflammation and activation of cell-mediated immunity in Myalgic Encephalomyelitis/Chronic Fatigue Syndrome (ME/CFS): Increased interleukin-1, tumor necrosis factor- $\alpha$, PMN-elastase, lysozyme and neopterin, J. Affect. Disord. 136 (2012) 933-939. doi:10.1016/J.JAD.2011.09.004.

[21] M. Maes, F.N.M. Twisk, M. Kubera, K. Ringel, J.C. Leunis, M. Geffard, Increased IgA responses to the LPS of commensal bacteria is associated with inflammation and activation of cell-mediated immunity in chronic fatigue syndrome, J. Affect. Disord. (2012). doi:10.1016/j.jad.2011.09.010.

[22] M. Maes, M. Kubera, J.-C. Leunis, M. Berk, Increased IgA and IgM responses against gut commensals in chronic depression: further evidence for increased bacterial translocation or leaky gut., J. Affect. Disord. 141 (2012) 55-62. doi:10.1016/j.jad.2012.02.023.

[23] M.E. Harrington, Neurobiological studies of fatigue, Prog. Neurobiol. 99 (2012) 93-105. doi:10.1016/j.pneurobio.2012.07.004. 
[24] M.E. Roerink, M.E. van der Schaaf, C.A. Dinarello, H. Knoop, J.W.M. van der Meer, Interleukin-1 as a mediator of fatigue in disease: A narrative review, J. Neuroinflammation. 14 (2017) 1-21. doi:10.1186/s12974-017-0796-7.

[25] A. Sierra, F. de Castro, J. del Río-Hortega, J. Rafael Iglesias-Rozas, M. Garrosa, H. Kettenmann, The "Big-Bang" for modern glial biology: Translation and comments on Pío del Río-Hortega 1919 series of papers on microglia, Glia. 64 (2016) 1801-1840. doi:10.1002/glia.23046.

[26] I.M. Samokhvalov, N.I. Samokhvalova, S. Nishikawa, Cell tracing shows the contribution of the yolk sac to adult haematopoiesis, Nature. 446 (2007) 1056-1061. doi:10.1038/nature05725.

[27] F. Ginhoux, M. Greter, M. Leboeuf, S. Nandi, P. See, S. Gokhan, M.F. Mehler, S.J. Conway, L.G. Ng, E.R. Stanley, I.M. Samokhvalov, M. Merad, Fate Mapping Analysis Reveals That Adult Microglia Derive from Primitive Macrophages, Science (80-. ). 330 (2010) 841-845. doi:10.1126/science.1194637.

[28] M.L. Dubbelaar, L. Kracht, B.J.L. Eggen, E.W.G.M. Boddeke, The Kaleidoscope of Microglial Phenotypes, Front. Immunol. 9 (2018). doi:10.3389/fimmu.2018.01753.

[29] L.J. Lawson, V.H. Perry, P. Dri, S. Gordon, Heterogeneity in the distribution and morphology of microglia in the normal adult mouse brain., Neuroscience. 39 (1990) 15170.

[30] M. Olah, K. Biber, J. Vinet, H.W.G.M. Boddeke, Microglia phenotype diversity., CNS Neurol. Disord. Drug Targets. 10 (2011) 108-18.

[31] C. Hierro-Bujalance, B.J. Bacskai, M. Garcia-Alloza, In Vivo Imaging of Microglia With Multiphoton Microscopy., Front. Aging Neurosci. 10 (2018) 218. doi:10.3389/fnagi.2018.00218.

[32] S. Bok, T. Wang, C.-J. Lee, S.-U. Jeon, Y.-E. Kim, J. Kim, B.-J. Hong, C.J. Yoon, S. Kim, S.-H. Lee, H.J. Kim, I.H. Kim, K.H. Kim, G.-O. Ahn, In vivo imaging of activated microglia 
in a mouse model of focal cerebral ischemia by two-photon microscopy., Biomed. Opt. Express. 6 (2015) 3303-12. doi:10.1364/BOE.6.003303.

[33] A.M. Fontainhas, M. Wang, K.J. Liang, S. Chen, P. Mettu, M. Damani, R.N. Fariss, W. Li, W.T. Wong, Microglial Morphology and Dynamic Behavior Is Regulated by lonotropic Glutamatergic and GABAergic Neurotransmission, PLoS One. 6 (2011) e15973. doi:10.1371/journal.pone.0015973.

[34] D. Davalos, J. Grutzendler, G. Yang, J. V Kim, Y. Zuo, S. Jung, D.R. Littman, M.L. Dustin, W.-B. Gan, ATP mediates rapid microglial response to local brain injury in vivo, Nat. Neurosci. 8 (2005) 752-758. doi:10.1038/nn1472.

[35] S.E. Haynes, G. Hollopeter, G. Yang, D. Kurpius, M.E. Dailey, W.-B. Gan, D. Julius, The P2Y12 receptor regulates microglial activation by extracellular nucleotides, Nat. Neurosci. 9 (2006) 1512-1519. doi:10.1038/nn1805.

[36] S.T. Dheen, C. Kaur, E.-A. Ling, Microglial activation and its implications in the brain diseases., Curr. Med. Chem. 14 (2007) 1189-97.

[37] R.M. Ransohoff, A polarizing question: do M1 and M2 microglia exist?, Nat. Neurosci. 19 (2016) 987-991. doi:10.1038/nn.4338.

[38] R. Yirmiya, N. Rimmerman, R. Reshef, Depression as a Microglial Disease, Trends Neurosci. 38 (2015) 637-658. doi:10.1016/j.tins.2015.08.001.

[39] H. Wake, A.J. Moorhouse, S. Jinno, S. Kohsaka, J. Nabekura, Resting Microglia Directly Monitor the Functional State of Synapses In Vivo and Determine the Fate of Ischemic Terminals, J. Neurosci. 29 (2009) 3974-3980. doi:10.1523/JNEUROSCI.4363-08.2009.

[40] M.-E. Tremblay, R.L. Lowery, A.K. Majewska, Microglial Interactions with Synapses Are Modulated by Visual Experience, PLoS Biol. 8 (2010) e1000527. doi:10.1371/journal.pbio.1000527.

[41] H. Kettenmann, F. Kirchhoff, A. Verkhratsky, Microglia: New Roles for the Synaptic Stripper, Neuron. 77 (2013) 10-18. doi:10.1016/j.neuron.2012.12.023. 
[42] U.B. Eyo, J. Peng, P. Swiatkowski, A. Mukherjee, A. Bispo, L.-J. Wu, Neuronal Hyperactivity Recruits Microglial Processes via Neuronal NMDA Receptors and Microglial P2Y12 Receptors after Status Epilepticus, J. Neurosci. 34 (2014) 10528-10540. doi:10.1523/JNEUROSCI.0416-14.2014.

[43] E.S. Wohleb, Neuron - Microglia interactions in Mental Health Disorders : " For Better , and For worse ," 7 (2016) 1-13. doi:10.3389/fimmu.2016.00544.

[44] A. Sierra, J.M. Encinas, J.J.P. Deudero, J.H. Chancey, G. Enikolopov, L.S. OverstreetWadiche, S.E. Tsirka, M. Maletic-Savatic, Microglia Shape Adult Hippocampal Neurogenesis through Apoptosis-Coupled Phagocytosis, Cell Stem Cell. 7 (2010) 483495. doi:10.1016/j.stem.2010.08.014.

[45] G.K. Sheridan, K.J. Murphy, Neuron-glia crosstalk in health and disease: fractalkine and CX3CR1 take centre stage., Open Biol. 3 (2013) 130181. doi:10.1098/rsob.130181.

[46] O. Pascual, S. Ben Achour, P. Rostaing, A. Triller, A. Bessis, Microglia activation triggers astrocyte-mediated modulation of excitatory neurotransmission, Proc. Natl. Acad. Sci. 109 (2012) E197-E205. doi:10.1073/pnas.1111098109.

[47] M. Domercq, L. Brambilla, E. Pilati, J. Marchaland, A. Volterra, P. Bezzi, P2Y1 Receptorevoked Glutamate Exocytosis from Astrocytes, J. Biol. Chem. 281 (2006) 30684-30696. doi:10.1074/jbc.M606429200.

[48] R. Orihuela, C.A. McPherson, G.J. Harry, Microglial M1/M2 polarization and metabolic states, Br. J. Pharmacol. 173 (2016) 649-665. doi:10.1111/bph.13139.

[49] M.G. Netea, F.L. van de Veerdonk, J.W.M. van der Meer, C.A. Dinarello, L.A.B. Joosten, Inflammasome-Independent Regulation of IL-1-Family Cytokines, Annu. Rev. Immunol. 33 (2015) 49-77. doi:10.1146/annurev-immunol-032414-112306.

[50] F. Martinon, A. Mayor, J. Tschopp, The Inflammasomes: Guardians of the Body, Annu. Rev. Immunol. 27 (2009) 229-265. doi:10.1146/annurev.immunol.021908.132715.

[51] J. Roth, G.E.P. de Souza, Fever induction pathways: evidence from responses to 
systemic or local cytokine formation, Brazilian J. Med. Biol. Res. 34 (2001) 301-314. doi:10.1590/S0100-879X2001000300003.

[52] T. Cartmell, G.N. Luheshi, N.J. Rothwell, Brain sites of action of endogenous interleukin-1 in the febrile response to localized inflammation in the rat., J. Physiol. 518 ( Pt 2 (1999) 585-94. doi:10.1111/J.1469-7793.1999.0585P.X.

[53] K.A. Jewett, J.M. Krueger, Humoral sleep regulation; interleukin-1 and tumor necrosis factor., Vitam. Horm. 89 (2012) 241-57. doi:10.1016/B978-0-12-394623-2.00013-5.

[54] M.R. Opp, L.A. Toth, Somnogenic and pyrogenic effects of interleukin-1beta and lipopolysaccharide in intact and vagotomized rats., Life Sci. 62 (1998) 923-36.

[55] M.D. Olivadoti, M.R. Opp, Effects of i.c.v. administration of interleukin-1 on sleep and body temperature of interleukin-6-deficient mice., Neuroscience. 153 (2008) 338-48. doi:10.1016/j.neuroscience.2008.02.008.

[56] A.H. Miller, E. Haroon, C.L. Raison, J.C. Felger, Cytokine targets in the brain: impact on neurotransmitters and neurocircuits., Depress. Anxiety. 30 (2013) 297-306. doi:10.1002/da.22084.

[57] E. Vereker, E. O'Donnell, M.A. Lynch, The inhibitory effect of interleukin-1beta on longterm potentiation is coupled with increased activity of stress-activated protein kinases., J. Neurosci. 20 (2000) 6811-9.

[58] R.M. Barrientos, M.G. Frank, A.M. Hein, E.A. Higgins, L.R. Watkins, J.W. Rudy, S.F. Maier, Time course of hippocampal IL-1 beta and memory consolidation impairments in aging rats following peripheral infection., Brain. Behav. Immun. 23 (2009) 46-54. doi:10.1016/j.bbi.2008.07.002.

[59] M. Monif, C.A. Reid, K.L. Powell, K.J. Drummond, T.J. O’Brien, D.A. Williams, Interleukin$1 \beta$ has trophic effects in microglia and its release is mediated by P2X7R pore, $\mathrm{J}$. Neuroinflammation. 13 (2016) 173. doi:10.1186/s12974-016-0621-8.

[60] X. Liu, N. Quan, Microglia and CNS interleukin-1: Beyond immunological concepts, Front. 
Neurol. 9 (2018) 1-11. doi:10.3389/fneur.2018.00008.

[61] I. Goshen, R. Yirmiya, K. Iverfeldt, J. Weidenfeld, The Role of Endogenous Interleukin-1 in Stress-Induced Adrenal Activation and Adrenalectomy-Induced Adrenocorticotropic Hormone Hypersecretion, Endocrinology. 144 (2003) 4453-4458. doi:10.1210/en.20030338.

[62] J.S.D. Winter, K.W. GOW, Y.S. PERRY, A.H. GREENBERG, A Stimulatory Effect of Interleukin-1 on Adrenocortical Cortisol Secretion Mediated by Prostaglandins*, Endocrinology. 127 (1990) 1904-1909. doi:10.1210/endo-127-4-1904.

[63] H. Schneider, F. Pitossi, D. Balschun, A. Wagner, A. del Rey, H.O. Besedovsky, A neuromodulatory role of interleukin-1 $\beta$ in the hippocampus, Proc. Natl. Acad. Sci. 95 (1998) 7778 LP-7783. doi:10.1073/pnas.95.13.7778.

[64] R. Yirmiya, I. Goshen, Immune modulation of learning, memory, neural plasticity and neurogenesis., Brain. Behav. Immun. 25 (2011) 181-213. doi:10.1016/j.bbi.2010.10.015.

[65] J.L. Remus, R. Dantzer, Inflammation models of depression in rodents: Relevance to psychotropic drug discovery, Int. J. Neuropsychopharmacol. 19 (2016) 1-13. doi:10.1093/ijnp/pyw028.

[66] M. Maes, M. Kubera, J.C. Leunis, The gut-brain barrier in major depression: intestinal mucosal dysfunction with an increased translocation of LPS from gram negative enterobacteria (leaky gut) plays a role in the inflammatory pathophysiology of depression., Neuro Endocrinol. Lett. (2008). doi:NEL290108A12 [pii].

[67] S. Biesmans, T.F. Meert, J.A. Bouwknecht, P.D. Acton, N. Davoodi, P. De Haes, J. Kuijlaars, X. Langlois, L.J.R. Matthews, L. Ver Donck, N. Hellings, R. Nuydens, Systemic immune activation leads to neuroinflammation and sickness behavior in mice., Mediators Inflamm. 2013 (2013) 271359. doi:10.1155/2013/271359.

[68] C.S. Custódio, B.S.F. Mello, R.C. Cordeiro, F.Y.R. de Araújo, J.H. Chaves, S.M.M. Vasconcelos, H.V. Nobre Júnior, F.C.F. de Sousa, M.L. Vale, A.F. Carvalho, D.S. 
Macêdo, Time course of the effects of lipopolysaccharide on prepulse inhibition and brain nitrite content in mice., Eur. J. Pharmacol. 713 (2013) 31-8. doi:10.1016/j.ejphar.2013.04.040.

[69] K.C. Cai, S. van Mil, E. Murray, J.-F. Mallet, C. Matar, N. Ismail, Age and sex differences in immune response following LPS treatment in mice, Brain. Behav. Immun. 58 (2016) 327-337. doi:10.1016/j.bbi.2016.08.002.

[70] A.W. Corona, D.M. Norden, J.P. Skendelas, Y. Huang, J.C. O'Connor, M. Lawson, R. Dantzer, K.W. Kelley, J.P. Godbout, Indoleamine 2,3-dioxygenase inhibition attenuates lipopolysaccharide induced persistent microglial activation and depressive-like complications in fractalkine receptor (CX(3)CR1)-deficient mice., Brain. Behav. Immun. 31 (2013) 134-42. doi:10.1016/j.bbi.2012.08.008.

[71] A.M. Fenn, J.C. Gensel, Y. Huang, P.G. Popovich, J. Lifshitz, J.P. Godbout, Immune Activation Promotes Depression 1 Month After Diffuse Brain Injury: A Role for Primed Microglia, Biol. Psychiatry. 76 (2014) 575-584. doi:10.1016/j.biopsych.2013.10.014.

[72] B.S.F. Mello, A.S. Monte, R.S. Mclntyre, J.K. Soczynska, C.S. Custódio, R.C. Cordeiro, J.H. Chaves, S.M.M. Vasconcelos, H.V. Nobre, F.C. Florenço de Sousa, T.N. Hyphantis, A.F. Carvalho, D.S. Macêdo, Effects of doxycycline on depressive-like behavior in mice after lipopolysaccharide (LPS) administration., J. Psychiatr. Res. 47 (2013) 1521-9. doi:10.1016/j.jpsychires.2013.06.008.

[73] E. Furube, S. Kawai, H. Inagaki, S. Takagi, S. Miyata, Brain Region-dependent Heterogeneity and Dose-dependent Difference in Transient Microglia Population Increase during Lipopolysaccharide-induced Inflammation, Sci. Rep. 8 (2018) 2203. doi:10.1038/s41598-018-20643-3.

[74] F.T.S. Rodrigues, M.R.M. de Souza, C.N. de C. Lima, F.E.R. da Silva, D.V. da S. Costa, C.C. dos Santos, F. Miyajima, F.C.F. de Sousa, S.M.M. Vasconcelos, T. Barichello, J. Quevedo, M. Maes, D.F. de Lucena, D. Macedo, Major depression model induced by 
repeated and intermittent lipopolysaccharide administration: Long-lasting behavioral, neuroimmune and neuroprogressive alterations, J. Psychiatr. Res. 107 (2018) 57-67. doi:10.1016/j.jpsychires.2018.10.003.

[75] S. Sugama, T. Takenouchi, M. Fujita, B. Conti, M. Hashimoto, Differential microglial activation between acute stress and lipopolysaccharide treatment, J. Neuroimmunol. 207 (2009) 24-31. doi:10.1016/j.jneuroim.2008.11.007.

[76] S. Sarkar, M. V. Zaretskaia, D. V. Zaretsky, M. Moreno, J.A. DiMicco, Stress- and lipopolysaccharide-induced c-fos expression and nNOS in hypothalamic neurons projecting to medullary raphe in rats: a triple immunofluorescent labeling study, Eur. J. Neurosci. 26 (2007) 2228-2238. doi:10.1111/j.1460-9568.2007.05843.x.

[77] F.R. Walker, M. Nilsson, K. Jones, Acute and chronic stress-induced disturbances of microglial plasticity, phenotype and function., Curr. Drug Targets. 14 (2013) 1262-76. doi:10.2174/13894501113149990208.

[78] M.-S. Kwon, Y.-J. Seo, J.-K. Lee, H.-K. Lee, J.-S. Jung, J.-E. Jang, S.-H. Park, H.-W. Suh, The repeated immobilization stress increases IL-1 $\beta$ immunoreactivities in only neuron, but not astrocyte or microglia in hippocampal CA1 region, striatum and paraventricular nucleus, Neurosci. Lett. 430 (2008) 258-263. doi:10.1016/j.neulet.2007.11.006.

[79] A. Toyoda, Social defeat models in animal science: What we have learned from rodent models, Anim. Sci. J. 88 (2017) 944-952. doi:10.1111/asj.12809.

[80] M.T. Bailey, H. Engler, N.D. Powell, D.A. Padgett, J.F. Sheridan, Repeated social defeat increases the bactericidal activity of splenic macrophages through a Toll-like receptordependent pathway., Am. J. Physiol. Regul. Integr. Comp. Physiol. 293 (2007) R1180-90. doi:10.1152/ajpregu.00307.2007.

[81] K. Ataka, A. Asakawa, K. Nagaishi, K. Kaimoto, A. Sawada, Y. Hayakawa, R. Tatezawa, A. Inui, M. Fujimiya, Bone marrow-derived microglia infiltrate into the paraventricular 
nucleus of chronic psychological stress-loaded mice., PLoS One. 8 (2013) e81744. doi:10.1371/journal.pone.0081744.

[82] E.S. Wohleb, A.M. Fenn, A.M. Pacenta, N.D. Powell, J.F. Sheridan, J.P. Godbout, Peripheral innate immune challenge exaggerated microglia activation, increased the number of inflammatory CNS macrophages, and prolonged social withdrawal in socially defeated mice, Psychoneuroendocrinology. 37 (2012) 1491-1505. doi:10.1016/j.psyneuen.2012.02.003.

[83] M. Hinwood, R.J. Tynan, J.L. Charnley, S.B. Beynon, T.A. Day, F.R. Walker, Chronic Stress Induced Remodeling of the Prefrontal Cortex: Structural Re-Organization of Microglia and the Inhibitory Effect of Minocycline, Cereb. Cortex. 23 (2013) 1784-1797. doi:10.1093/cercor/bhs151.

[84] R.J. Tynan, S. Naicker, M. Hinwood, E. Nalivaiko, K.M. Buller, D. V. Pow, T.A. Day, F.R. Walker, Chronic stress alters the density and morphology of microglia in a subset of stress-responsive brain regions, Brain. Behav. Immun. 24 (2010) 1058-1068. doi:10.1016/j.bbi.2010.02.001.

[85] M.G. Frank, B.M. Thompson, L.R. Watkins, S.F. Maier, Glucocorticoids mediate stressinduced priming of microglial pro-inflammatory responses, Brain. Behav. Immun. 26 (2012) 337-345. doi:10.1016/j.bbi.2011.10.005.

[86] M.A. Calcia, D.R. Bonsall, P.S. Bloomfield, S. Selvaraj, T. Barichello, O.D. Howes, Stress and neuroinflammation: a systematic review of the effects of stress on microglia and the implications for mental illness., Psychopharmacology (Berl). 233 (2016) 1637-50. doi:10.1007/s00213-016-4218-9.

[87] S. Roque, A.R. Mesquita, J.A. Palha, N. Sousa, M. Correia-Neves, The Behavioral and Immunological Impact of Maternal Separation: A Matter of Timing, Front. Behav. Neurosci. 8 (2014) 1-10. doi:10.3389/fnbeh.2014.00192.

[88] C.P. Carr, C.M.S. Martins, A.M. Stingel, V.B. Lemgruber, M.F. Juruena, The role of early 
life stress in adult psychiatric disorders: a systematic review according to childhood trauma subtypes., J. Nerv. Ment. Dis. 201 (2013) 1007-20.

doi:10.1097/NMD.0000000000000049.

[89] S.M. O’Mahony, J.R. Marchesi, P. Scully, C. Codling, A.-M. Ceolho, E.M.M. Quigley, J.F. Cryan, T.G. Dinan, Early life stress alters behavior, immunity, and microbiota in rats: implications for irritable bowel syndrome and psychiatric illnesses., Biol. Psychiatry. 65 (2009) 263-7. doi:10.1016/j.biopsych.2008.06.026.

[90] A. Roque, A. Ochoa-Zarzosa, L. Torner, Maternal separation activates microglial cells and induces an inflammatory response in the hippocampus of male rat pups, independently of hypothalamic and peripheral cytokine levels., Brain. Behav. Immun. 55 (2016) 39-48. doi:10.1016/j.bbi.2015.09.017.

[91] Y. Takatsuru, J. Nabekura, T. Ishikawa, S. Kohsaka, N. Koibuchi, Early-life stress increases the motility of microglia in adulthood, J. Physiol. Sci. 65 (2015) 187-194. doi:10.1007/s12576-015-0361-z.

[92] P. Ganguly, H.C. Brenhouse, Broken or maladaptive? Altered trajectories in neuroinflammation and behavior after early life adversity, Dev. Cogn. Neurosci. 11 (2014) 18-30. doi:10.1016/j.dcn.2014.07.001.

[93] M. Maes, Cytokines, Stress, and Depression, Springer US, Boston, MA, 1999. doi:10.1007/b102345.

[94] G. a W. Rook, C.L. Raison, C. a. Lowry, Can we vaccinate against depression?, Drug Discov. Today. 17 (2012) 451-458. doi:10.1016/j.drudis.2012.03.018.

[95] S.S. Coughlin, Anxiety and Depression: Linkages with Viral Diseases., Public Health Rev. 34 (2012).

[96] W. Zou, R. Feng, Y. Yang, Changes in the serum levels of inflammatory cytokines in antidepressant drug-naïve patients with major depression, PLoS One. 13 (2018) e0197267. doi:10.1371/journal.pone.0197267. 
[97] C.A. Köhler, T.H. Freitas, B. Stubbs, M. Maes, M. Solmi, N. Veronese, N.Q. de Andrade, G. Morris, B.S. Fernandes, A.R. Brunoni, N. Herrmann, C.L. Raison, B.J. Miller, K.L. Lanctôt, A.F. Carvalho, Peripheral Alterations in Cytokine and Chemokine Levels After Antidepressant Drug Treatment for Major Depressive Disorder: Systematic Review and Meta-Analysis, Mol. Neurobiol. (2017) 1-12. doi:10.1007/s12035-017-0632-1.

[98] C.M. Sandiego, J.-D. Gallezot, B. Pittman, N. Nabulsi, K. Lim, S.-F. Lin, D. Matuskey, J.Y. Lee, K.C. O'Connor, Y. Huang, R.E. Carson, J. Hannestad, K.P. Cosgrove, Imaging robust microglial activation after lipopolysaccharide administration in humans with PET., Proc. Natl. Acad. Sci. U. S. A. 112 (2015) 12468-73. doi:10.1073/pnas.1511003112.

[99] T.A. Bayer, R. Buslei, L. Havas, P. Falkai, Evidence for activation of microglia in patients with psychiatric illnesses., Neurosci. Lett. 271 (1999) 126-8.

[100] J. Hannestad, N. DellaGioia, J.-D. Gallezot, K. Lim, N. Nabulsi, I. Esterlis, B. Pittman, J.Y. Lee, K.C. O'Connor, D. Pelletier, R.E. Carson, The neuroinflammation marker translocator protein is not elevated in individuals with mild-to-moderate depression: $\mathrm{A}$ [11C]PBR28 PET study, Brain. Behav. Immun. 33 (2013) 131-138. doi:10.1016/j.bbi.2013.06.010.

[101] E. Setiawan, S. Attwells, A.A. Wilson, R. Mizrahi, P.M. Rusjan, L. Miler, C. Xu, S. Sharma, S. Kish, S. Houle, J.H. Meyer, Association of translocator protein total distribution volume with duration of untreated major depressive disorder: a crosssectional study., The Lancet. Psychiatry. 5 (2018) 339-347. doi:10.1016/S22150366(18)30048-8.

[102] E.M. Richards, P. Zanotti-Fregonara, M. Fujita, L. Newman, C. Farmer, E.D. Ballard, R. Machado-Vieira, P. Yuan, M.J. Niciu, C.H. Lyoo, I.D. Henter, G. Salvadore, W.C. Drevets, H. Kolb, R.B. Innis, C.A. Zarate Jr, PET radioligand binding to translocator protein (TSPO) is increased in unmedicated depressed subjects, EJNMMI Res. 8 (2018) 57. doi:10.1186/s13550-018-0401-9. 
[103] T.P. Schnieder, I. Trencevska, G. Rosoklija, A. Stankov, J.J. Mann, J. Smiley, A.J. Dwork, Microglia of prefrontal white matter in suicide., J. Neuropathol. Exp. Neurol. 73 (2014) 880-90. doi:10.1097/NEN.0000000000000107.

[104] J. Steiner, H. Bielau, R. Brisch, P. Danos, O. Ullrich, C. Mawrin, H.-G. Bernstein, B. Bogerts, Immunological aspects in the neurobiology of suicide: Elevated microglial density in schizophrenia and depression is associated with suicide, J. Psychiatr. Res. 42 (2008) 151-157. doi:10.1016/j.jpsychires.2006.10.013.

[105] S.G. Torres-Platas, C. Cruceanu, G.G. Chen, G. Turecki, N. Mechawar, Evidence for increased microglial priming and macrophage recruitment in the dorsal anterior cingulate white matter of depressed suicides, Brain. Behav. Immun. 42 (2014) 50-59. doi:10.1016/j.bbi.2014.05.007.

[106] S.E. Holmes, R. Hinz, S. Conen, C.J. Gregory, J.C. Matthews, J.M. Anton-Rodriguez, A. Gerhard, P.S. Talbot, Elevated Translocator Protein in Anterior Cingulate in Major Depression and a Role for Inflammation in Suicidal Thinking: A Positron Emission Tomography Study, Biol. Psychiatry. 83 (2018) 61-69.

doi:10.1016/j.biopsych.2017.08.005.

[107] T. Barichello, L.R. Simões, A. Collodel, V. V. Giridharan, F. Dal-Pizzol, D. Macedo, J. Quevedo, The translocator protein $(18 \mathrm{kDa})$ and its role in neuropsychiatric disorders, Neurosci. Biobehav. Rev. 83 (2017) 183-199. doi:10.1016/j.neubiorev.2017.10.010.

[108] G. Morris, M. Berk, K. Walder, M. Maes, Central pathways causing fatigue in neuroinflammatory and autoimmune illnesses, BMC Med. (2015). doi:10.1186/s12916-0140259-2.

[109] G. Morris, M. Berk, P. Galecki, K. Walder, M. Maes, The Neuro-Immune Pathophysiology of Central and Peripheral Fatigue in Systemic Immune-Inflammatory and Neuro-Immune Diseases, Mol. Neurobiol. (2016). doi:10.1007/s12035-015-9090-9.

[110] G. Morris, G. Anderson, P. Galecki, M. Berk, M. Maes, A narrative review on the 
similarities and dissimilarities between myalgic encephalomyelitis/chronic fatigue syndrome (ME/CFS) and sickness behavior, BMC Med. 11 (2013) 64. doi:10.1186/17417015-11-64.

[111] M. Maes, M. Berk, L. Goehler, C. Song, G. Anderson, P. Ga, Depression and sickness behavior are Janus-faced responses to shared inflammatory pathways, (2012) 1-19. doi:10.1186/1741-7015-10-66.

[112] D. Racciatti, M.T. Guagnano, J. Vecchiet, P.L. De Remigis, E. Pizzigallo, R. Della Vecchia, T. Di Sciascio, D. Merlitti, S. Sensi, Chronic Fatigue Syndrome: Circadian Rhythm and Hypothalamic-Pituitary-Adrenal (HPA) Axis Impairment, Int. J. Immunopathol. Pharmacol. 14 (2001) 11-15. doi:10.1177/039463200101400103.

[113] M.E. Harrington, N. Program, S. College, Neurobiological studies of fatigue, 99 (2013) 93-105. doi:10.1016/j.pneurobio.2012.07.004.Neurobiological.

[114] G.B. Lundkvist, R.H. Hill, K. Kristensson, Disruption of circadian rhythms in synaptic activity of the suprachiasmatic nuclei by African trypanosomes and cytokines., Neurobiol. Dis. 11 (2002) 20-7.

[115] J.W. Stiller, T.T. Postolache, Sleep-wake and Other Biological Rhythms: Functional Neuroanatomy, Clin. Sports Med. 24 (2005) 205-235. doi:10.1016/j.csm.2004.12.008.

[116] A.M. Rosenwasser, Functional neuroanatomy of sleep and circadian rhythms, Brain Res. Rev. 61 (2009) 281-306. doi:10.1016/j.brainresrev.2009.08.001.

[117] R. Benca, M.J. Duncan, E. Frank, C. McClung, R.J. Nelson, A. Vicentic, Biological rhythms, higher brain function, and behavior: Gaps, opportunities, and challenges, Brain Res. Rev. 62 (2009) 57-70. doi:10.1016/j.brainresrev.2009.09.005.

[118] A.H. Luo, G. Aston-Jones, Circuit projection from suprachiasmatic nucleus to ventral tegmental area: a novel circadian output pathway, Eur. J. Neurosci. 29 (2009) 748-760. doi:10.1111/j.1460-9568.2008.06606.x.

[119] S. Ohdo, S. Koyanagi, H. Suyama, S. Higuchi, H. Aramaki, Changing the dosing 
schedule minimizes the disruptive effects of interferon on clock function, Nat. Med. 7 (2001) 356-360. doi:10.1038/85507.

[120] L. Marpegán, T.A. Bekinschtein, M.A. Costas, D.A. Golombek, Circadian responses to endotoxin treatment in mice, J. Neuroimmunol. 160 (2005) 102-109. doi:10.1016/j.jneuroim.2004.11.003.

[121] N. Paladino, M.J. Leone, S.A. Plano, D.A. Golombek, Paying the circadian toll: The circadian response to LPS injection is dependent on the Toll-like receptor 4 , J. Neuroimmunol. 225 (2010) 62-67. doi:10.1016/j.jneuroim.2010.04.015.

[122] M. Bentivoglio, X.H. Deng, M. Nygård, A. Sadki, K. Kristensson, The aging suprachiasmatic nucleus and cytokines: Functional, molecular, and cellular changes in rodents, Chronobiol. Int. 23 (2006) 437-449. doi:10.1080/07420520500545797.

[123] S. Huitron-Resendiz, M.C.G. Marcondes, C.T. Flynn, C.M.S. Lanigan, H.S. Fox, Effects of simian immunodeficiency virus on the circadian rhythms of body temperature and gross locomotor activity, Proc. Natl. Acad. Sci. 104 (2007) 15138-15143. doi:10.1073/pnas.0707171104.

[124] G.B. Lundkvist, J. Christenson, R.A. ElTayeb, Z.C. Peng, P. Grillner, J. Mhlanga, M. Bentivoglio, K. Kristensson, Altered neuronal activity rhythm and glutamate receptor expression in the suprachiasmatic nuclei of Trypanosoma brucei-infected rats., J. Neuropathol. Exp. Neurol. 57 (1998) 21-9.

[125] N. Cermakian, T. Lange, D. Golombek, D. Sarkar, A. Nakao, S. Shibata, G. Mazzoccoli, Crosstalk between the circadian clock circuitry and the immune system, Chronobiol. Int. 30 (2013) 870-888. doi:10.3109/07420528.2013.782315.

[126] S. Chianella, M. Semprevivo, Z.C. Peng, D. Zaccheo, M. Bentivoglio, G. Grassi-Zucconi, Microglia activation in a model of sleep disorder: an immunohistochemical study in the rat brain during Trypanosoma brucei infection., Brain Res. 832 (1999) 54-62.

[127] K. Figarella, N.L. Uzcategui, S. Mogk, K. Wild, P. Fallier-Becker, J.J. Neher, M. 
Duszenko, Morphological changes, nitric oxide production, and phagocytosis are triggered in vitro in microglia by bloodstream forms of Trypanosoma brucei, Sci. Rep. 8 (2018) 15002. doi:10.1038/s41598-018-33395-x.

[128] M. Noda, M. Ifuku, M.S. Hossain, T. Katafuchi, Glial Activation and Expression of the Serotonin Transporter in Chronic Fatigue Syndrome, Front. Psychiatry. 9 (2018) 589. doi:10.3389/fpsyt.2018.00589.

[129] M. Ifuku, S.M. Hossain, M. Noda, T. Katafuchi, Induction of interleukin-1 $\beta$ by activated microglia is a prerequisite for immunologically induced fatigue, Eur. J. Neurosci. 40 (2014) 3253-3263. doi:10.1111/ejn.12668.

[130] M. Yamato, Y. Tamura, A. Eguchi, S. Kume, Y. Miyashige, M. Nakano, Y. Watanabe, Y. Kataoka, Brain Interleukin-1 $\beta$ and the Intrinsic Receptor Antagonist Control Peripheral Toll-Like Receptor 3-Mediated Suppression of Spontaneous Activity in Rats, PLoS One. 9 (2014) e90950. doi:10.1371/journal.pone.0090950.

[131] K. Louati, F. Berenbaum, Fatigue in chronic inflammation - a link to pain pathways, Arthritis Res. Ther. 17 (2015) 1-10. doi:10.1186/s13075-015-0784-1.

[132] Y. Nakatomi, K. Mizuno, A. Ishii, Y. Wada, M. Tanaka, S. Tazawa, K. Onoe, S. Fukuda, J. Kawabe, K. Takahashi, Y. Kataoka, S. Shiomi, K. Yamaguti, M. Inaba, H. Kuratsune, Y. Watanabe, Neuroinflammation in Patients with Chronic Fatigue Syndrome/Myalgic Encephalomyelitis: An 11C-(R)-PK11195 PET Study, J. Nucl. Med. 55 (2014) 945-950. doi:10.2967/jnumed.113.131045.

[133] C.W. Gay, M.E. Robinson, S. Lai, A. O’Shea, J.G. Craggs, D.D. Price, R. Staud, Abnormal Resting-State Functional Connectivity in Patients with Chronic Fatigue Syndrome: Results of Seed and Data-Driven Analyses., Brain Connect. 6 (2016) 48-56. doi:10.1089/brain.2015.0366.

[134] Z.Y. Shan, K. Finegan, S. Bhuta, T. Ireland, D.R. Staines, S.M. Marshall-Gradisnik, L.R. Barnden, Decreased Connectivity and Increased Blood Oxygenation Level Dependent 
Complexity in the Default Mode Network in Individuals with Chronic Fatigue Syndrome, Brain Connect. 8 (2018) 33-39. doi:10.1089/brain.2017.0549.

[135] S. Blundell, K.K. Ray, M. Buckland, P.D. White, Chronic fatigue syndrome and circulating cytokines: A systematic review, Brain. Behav. Immun. 50 (2015) 186-195. doi:10.1016/j.bbi.2015.07.004.

[136] L. Russell, G. Broderick, R. Taylor, H. Fernandes, J. Harvey, Z. Barnes, A. Smylie, F. Collado, E.G. Balbin, B.Z. Katz, N.G. Klimas, M.A. Fletcher, Illness progression in chronic fatigue syndrome: a shifting immune baseline, BMC Immunol. 17 (2016) 3. doi:10.1186/s12865-016-0142-3.

[137] S.L. Hardcastle, E.W. Brenu, S. Johnston, T. Nguyen, T. Huth, S. Ramos, D. Staines, S. Marshall-Gradisnik, Serum Immune Proteins in Moderate and Severe Chronic Fatigue Syndrome/Myalgic Encephalomyelitis Patients, Int. J. Med. Sci. 12 (2015) 764-772. doi:10.7150/ijms.12399.

[138] A. Landi, D. Broadhurst, S.D. Vernon, D.L.J. Tyrrell, M. Houghton, Reductions in circulating levels of IL-16, IL-7 and VEGF-A in myalgic encephalomyelitis/chronic fatigue syndrome, Cytokine. 78 (2016) 27-36. doi:10.1016/j.cyto.2015.11.018.

[139] C.C. Chao, E.N. Janoff, S. Hu, K. Thomas, M. Gallagher, M. Tsang, P.K. Peterson, Altered cytokine release in peripheral blood mononuclear cell cultures from patients with the chronic fatigue syndrome, Cytokine. (1991). doi:10.1016/1043-4666(91)90497-2.

[140] A.C. Mawle, R. Nisenbaum, J.G. Dobbins, H.E. Gary, J.A. Stewart, M. Reyes, L. Steele, D.S. Schmid, W.C. Reeves, Immune responses associated with chronic fatigue syndrome: a case-control study., J. Infect. Dis. 175 (1997) 136-41.

[141] C.M. Swanink, J.H. Vercoulen, J.M. Galama, M.T. Roos, L. Meyaard, J. van der VenJongekrijg, R. de Nijs, G. Bleijenberg, J.F. Fennis, F. Miedema, J.W. van der Meer, Lymphocyte subsets, apoptosis, and cytokines in patients with chronic fatigue syndrome., J. Infect. Dis. 173 (1996) 460-3. 
[142] U. Vollmer-Conna, C. Fazou, B. Cameron, H. Li, C. Brennan, L. Luck, T. Davenport, D. Wakefield, I. Hickie, A. Lloyd, Production of pro-inflammatory cytokines correlates with the symptoms of acute sickness behaviour in humans., Psychol. Med. 34 (2004) 128997.

[143] V.-C. Ute, B. Cameron, D.H. Pavlovic, K. Singletary, T. Davenport, S. Vernon, W.C. Reeves, I. Hickie, D. Wakefield, A.R. Lloyd, Dubbo Infective Outcomes Study Group, Postinfective Fatigue Syndrome Is Not Associated with Altered Cytokine Production, Clin. Infect. Dis. 45 (2007) 732-735. doi:10.1086/520990.

[144] D. Peterson, E.W. Brenu, G. Gottschalk, S. Ramos, T. Nguyen, D. Staines, S. MarshallGradisnik, Cytokines in the Cerebrospinal Fluids of Patients with Chronic Fatigue Syndrome/Myalgic Encephalomyelitis, Mediators Inflamm. 2015 (2015) 1-4. doi:10.1155/2015/929720.

[145] B.H. Natelson, S.A. Weaver, C.-L. Tseng, J.E. Ottenweller, Spinal fluid abnormalities in patients with chronic fatigue syndrome., Clin. Diagn. Lab. Immunol. 12 (2005) 52-5. doi:10.1128/CDLI.12.1.52-55.2005.

[146] M. Hornig, G. Gottschalk, D.L. Peterson, K.K. Knox, A.F. Schultz, M.L. Eddy, X. Che, W.I. Lipkin, Cytokine network analysis of cerebrospinal fluid in myalgic encephalomyelitis / chronic fatigue syndrome, Mol. Psychiatry. 21 (2016) 261-269. doi:10.1038/mp.2015.29.

[147] J.G. Montoya, T.H. Holmes, J.N. Anderson, H.T. Maecker, Y. Rosenberg-Hasson, I.J. Valencia, L. Chu, J.W. Younger, C.M. Tato, M.M. Davis, Cytokine signature associated with disease severity in chronic fatigue syndrome patients., Proc. Natl. Acad. Sci. U. S. A. 114 (2017) E7150-E7158. doi:10.1073/pnas.1710519114.

[148] S.F. Milrad, D.L. Hall, D.R. Jutagir, E.G. Lattie, G.H. Ironson, W. Wohlgemuth, M.V. Nunez, L. Garcia, S.J. Czaja, D.M. Perdomo, M.A. Fletcher, N. Klimas, M.H. Antoni, Poor sleep quality is associated with greater circulating pro-inflammatory cytokines and severity and frequency of chronic fatigue syndrome/myalgic encephalomyelitis (CFS/ME) 
symptoms in women, J. Neuroimmunol. 303 (2017) 43-50.

doi:10.1016/j.jneuroim.2016.12.008.

[149] M. Maes, F.N.M. Twisk, K. Ringel, Inflammatory and Cell-Mediated Immune Biomarkers in Myalgic Encephalomyelitis/Chronic Fatigue Syndrome and Depression: Inflammatory Markers Are Higher in Myalgic Encephalomyelitis/Chronic Fatigue Syndrome than in Depression, Psychother. Psychosom. 81 (2012) 286-295. doi:10.1159/000336803.

[150] M. Maes, K. Ringel, M. Kubera, G. Anderson, G. Morris, P. Galecki, M. Geffard, In myalgic encephalomyelitis/chronic fatigue syndrome, increased autoimmune activity against 5-HT is associated with immuno-inflammatory pathways and bacterial translocation, J. Affect. Disord. 150 (2013) 223-230. doi:10.1016/j.jad.2013.03.029.

[151] R. Klein, P.A. Berg, High incidence of antibodies to 5-hydroxytryptamine, gangliosides and phospholipids in patients with chronic fatigue and fibromyalgia syndrome and their relatives: evidence for a clinical entity of both disorders., Eur. J. Med. Res. 1 (1995) 21-6.

[152] M. Nishikai, S. Tomomatsu, R.W. Hankins, S. Takagi, K. Miyachi, S. Kosaka, K. Akiya, Autoantibodies to a $68 / 48 \mathrm{kDa}$ protein in chronic fatigue syndrome and primary fibromyalgia: a possible marker for hypersomnia and cognitive disorders., Rheumatology (Oxford). 40 (2001) 806-10.

[153] G. Morris, M. Berk, P. Galecki, M. Maes, The emerging role of autoimmunity in myalgic encephalomyelitis/chronic fatigue syndrome (ME/cfs), Mol. Neurobiol. 49 (2014) 741756. doi:10.1007/s12035-013-8553-0.

[154] M. Jokela, M. Virtanen, G.D. Batty, M. Kivimäki, Inflammation and Specific Symptoms of Depression, JAMA Psychiatry. 73 (2016) 87. doi:10.1001/jamapsychiatry.2015.1977.

[155] A.L. Chu, J. Stochl, G. Lewis, S. Zammit, P.B. Jones, G.M. Khandaker, Longitudinal association between inflammatory markers and specific symptoms of depression in a prospective birth cohort, Brain. Behav. Immun. (2018) 1-8.

doi:10.1016/j.bbi.2018.11.007. 
[156] Y.-M. Bai, W.-F. Chiou, T.-P. Su, C.-T. Li, M.-H. Chen, Pro-inflammatory cytokine associated with somatic and pain symptoms in depression, J. Affect. Disord. 155 (2014) 28-34. doi:10.1016/j.jad.2013.10.019.

[157] F. Euteneuer, M.J. Schwarz, K. Dannehl, A. Hartung, S. Westermann, W. Rief, Increased soluble interleukin-2 receptor levels are related to somatic but not to cognitive-affective features in major depression, Brain. Behav. Immun. 26 (2012) 1244-1248. doi:10.1016/j.bbi.2012.06.007.

[158] C. Roomruangwong, B. Kanchanatawan, S. Sirivichayakul, G. Anderson, A.F. Carvalho, S. Duleu, M. Geffard, M. Maes, IgA/lgM responses to tryptophan and tryptophan catabolites (TRYCATs) are differently associated with prenatal depression, physiosomatic symptoms at the end of term and premenstrual syndrome, Mol. Neurobiol. 54 (2017) 3038-3049. doi:10.1007/s12035-016-9877-3.

[159] K. Dannehl, W. Rief, M.J. Schwarz, A. Hennings, S. Riemer, V. Selberdinger, T. Stapf, F. Euteneuer, The predictive value of somatic and cognitive depressive symptoms for cytokine changes in patients with major depression, Neuropsychiatr. Dis. Treat. 10 (2014) 1191. doi:10.2147/NDT.S61640.

[160] C.L. Raison, J.-M.S. Lin, W.C. Reeves, Association of peripheral inflammatory markers with chronic fatigue in a population-based sample, Brain. Behav. Immun. 23 (2009) 327337. doi:10.1016/j.bbi.2008.11.005.

[161] N. Groven, E.A. Fors, V.C. Iversen, L.R. White, S.K. Reitan, Association between cytokines and psychiatric symptoms in chronic fatigue syndrome and healthy controls, Nord. J. Psychiatry. (2018) 1-5. doi:10.1080/08039488.2018.1493747.

[162] S.F. Milrad, D.L. Hall, D.R. Jutagir, E.G. Lattie, S.J. Czaja, D.M. Perdomo, M.A. Fletcher, N. Klimas, M.H. Antoni, Depression, evening salivary cortisol and inflammation in chronic fatigue syndrome: A psychoneuroendocrinological structural regression model, Int. J. Psychophysiol. 131 (2018) 124-130. doi:10.1016/j.ijpsycho.2017.09.009. 
[163] M. Maes, P. Galecki, R. Verkerk, W. Rief, Somatization, but not depression, is characterized by disorders in the tryptophan catabolite (TRYCAT) pathway, indicating increased indoleamine 2,3-dioxygenase and lowered kynurenine aminotransferase activity., Neuro Endocrinol. Lett. 32 (2011) 264-73.

[164] M. Maes, An intriguing and hitherto unexplained co-occurrence: Depression and chronic fatigue syndrome are manifestations of shared inflammatory, oxidative and nitrosative (IO\&NS) pathways, Prog. Neuro-Psychopharmacology Biol. Psychiatry. (2011). doi:10.1016/j.pnpbp.2010.06.023. 